\title{
The resultant on compact Riemann surfaces
}

\author{
Björn Gustafsson and Vladimir G. Tkachev
}

\begin{abstract}
We introduce a notion of resultant of two meromorphic functions on a compact Riemann surface and demonstrate its usefulness in several respects. For example, we exhibit several integral formulas for the resultant, relate it to potential theory and give explicit formulas for the algebraic dependence between two meromorphic functions on a compact Riemann surface. As a particular application, the exponential transform of a quadrature domain in the complex plane is expressed in terms of the resultant of two meromorphic functions on the Schottky double of the domain.
\end{abstract}

\section{Introduction}

A bounded domain $\Omega$ in the complex plane is called a (classical) quadrature domain $[\mathbf{1}],[\mathbf{4 2}],[\mathbf{4 6}],[\mathbf{2 6}]$ or, in a different terminology, an algebraic domain $[\mathbf{5 3}]$, if there exist finitely many points $z_{i} \in \Omega$ and coefficients $c_{k j} \in \mathbb{C}$ $(i=1, \ldots, N$, say $)$ such that

$$
\int_{\Omega} h d x d y=\sum_{k=1}^{N} \sum_{j=1}^{s_{k}} c_{k j} h^{(j-1)}\left(z_{k}\right)
$$

for every integrable analytic function $h$ in $\Omega[39]$. In the last two decades there has been a growing interest in the applications of quadrature domains to various problems in mathematics and theoretical physics, ranging from Laplacian growth to integrable systems and string theory (see recent articles $[\mathbf{2 5}]),[\mathbf{3 0}]$, and the references therein).

One of the most intriguing properties of quadrature domains is their algebraicity $[\mathbf{1}],[\mathbf{2 2}]$ : the boundary of a quadrature domain is (modulo finitely many points) the full real section of an algebraic curve:

$$
\partial \Omega=\{z \in \mathbb{C}: Q(z, \bar{z})=0\},
$$

where $Q(z, w)$ is an irreducible Hermitian polynomial. Moreover, the corresponding full algebraic curve (essentially $\left\{(z, w) \in \mathbb{C}^{2}: Q(z, w)=0\right\}$ ) can be naturally identified with the Schottky double $\widehat{\Omega}$ of $\Omega$ by means of the Schwarz function $S(z)$ of $\partial \Omega$. The latter satisfies $S(z)=\bar{z}$ on $\partial \Omega$ and is, in the case of a quadrature domain, meromorphic in all $\Omega$.

1991 Mathematics Subject Classification. 12E05, 14Q99, 30F10, 31A15, 47B35.

Key words and phrases. Resultant, Toeplitz operator, Weil reciprocity, Serre duality, exponential transform, quadrature domain, local symbol. 
A deep impact into the theory of quadrature domains was the discovery by M. Putinar [37] in the mid 1990's of an alternative characterization in terms of hyponormal operators. Recall that J. Pincus proved [34] that with any bounded linear operator $T: H \rightarrow H$ in a Hilbert space $H$ for which the self-commutator is positive (i.e., $T$ is hyponormal) and has rank one, say

$$
\left[T^{*}, T\right]=T^{*} T-T T^{*}=\xi \otimes \xi, \quad 0 \neq \xi \in H,
$$

one can associate a unitary invariant, the so-called principal function. This is a measurable function $g: \mathbb{C} \rightarrow[0,1]$, supported on the spectrum of $T$, such that for any $z, w$ in the resolvent set of $T$ there holds

$$
\operatorname{det}\left(T_{z}^{*} T_{w} T_{z}^{*-1} T_{w}{ }^{-1}\right)=\exp \left[\frac{1}{2 \pi \mathrm{i}} \int_{\mathbb{C}} \frac{g(\zeta) d \zeta \wedge d \bar{\zeta}}{(\zeta-z)(\bar{\zeta}-\bar{w})}\right]
$$

where $T_{u}=T-u I$. The right hand side in (3) is referred to as the exponential transform of the function $g$. In case $g$ is the characteristic function of a bounded set $\Omega$ we have the exponential transform of $\Omega$,

$$
E_{\Omega}(z, w)=\exp \left[\frac{1}{2 \pi \mathrm{i}} \int_{\Omega} \frac{d \zeta}{\zeta-z} \wedge \frac{d \bar{\zeta}}{\bar{\zeta}-\bar{w}}\right]
$$

A central result in Putinar's theory is the following criterion: a domain $\Omega$ is a quadrature domain if and only if the exponential transform of $\Omega$ is a rational function of the form

$$
E_{\Omega}(z, w)=\frac{Q(z, w)}{P(z) \overline{P(w)}}, \quad|z|,|w| \gg 1,
$$

where $P$ and $Q$ are polynomials. In this case $Q$ is the same as the polynomial in $(2)$.

In the present paper we shall unify the above pictures by interpreting the exponential transform of a quadrature domain in terms of resultants of meromorphic functions on the Schottky double of the domain. To this end we need to extend the classical concept of resultant of two polynomials to a notion of resultant for meromorphic functions on a compact Riemann surface. The introduction of such a meromorphic resultant and the demonstration of its usefulness in several contexts is the main overall purpose of this paper.

The definition of the resultant is natural and simple: given two meromorphic functions $f$ and $g$ on a compact Riemann surface $M$ we define their meromorphic resultant as

$$
\mathcal{R}(f, g)=\prod_{i=1}^{m} \frac{g\left(a_{i}\right)}{g\left(b_{i}\right)},
$$

where $(f)=\sum a_{i}-\sum b_{i}=f^{-1}(0)-f^{-1}(\infty)$ is the divisor of $f$. This resultant actually depends only on the divisors of $f$ and $g$. It follows from Weil's reciprocity law that the resultant is symmetric:

$$
\mathcal{R}(f, g)=\mathcal{R}(g, f) .
$$

For the genus zero case the meromorphic resultant is just a cross-ratio product of four polynomial resultants, whereas for higher genus surfaces it can be expressed as a cross-ratio product of values of theta functions. In the other direction, the classical resultant of two polynomials (which can 
be viewed as meromorphic functions with a marked pole) may be recovered from the meromorphic one by specifying a local symbol at the infinity (see Section 9).

It is advantageous in many contexts to amplify the resultant to an elimination function. With $f$ and $g$ as above this is defined as

$$
\mathcal{E}_{f, g}(z, w)=\mathcal{R}(f-z, g-w),
$$

where $z, w$ are free complex parameters. Thus defined, $\mathcal{E}_{f, g}(z, w)$ is a rational function in $z$ and $w$ having the elimination property

$$
\mathcal{E}_{f, g}(f(\zeta), g(\zeta))=0 \quad(\zeta \in M) .
$$

In particular, this gives an explicit formula for the algebraic dependence between two meromorphic functions on a compact Riemann surface. Treating the variables $z$ and $w$ in the definition of $\mathcal{E}_{f, g}(z, w)$ as spectral parameters in the elimination problem, the above identity resembles the Cayley-Hamilton theorem for the characteristic polynomial in linear algebra. This analogy becomes more clear by passing to the so-called differential resultant in connection with the spectral curves for two commutating ODE's, see for example [35].

The above aspects of the resultant and the elimination function characterize them essentially from an algebraic side. In the paper we shall however much emphasize the analytic point of view by relating the resultant to objects such as the exponential transform (4) and the Fredholm determinant. One of the key results is an integral representation of the resultant (Theorem 2), somewhat similar to (4). From this we deduce one of the main results of the paper: the exponential transform of a quadrature domain $\Omega$ coincides with a natural elimination function on the Schottky double $\widehat{\Omega}$ of $\Omega$ :

$$
E_{\Omega}(z, w)=\mathcal{E}_{f, f^{*}}(z, \bar{w}) .
$$

Here $\left(f, f^{*}\right)$ is a canonical pair of meromorphic functions on $\widehat{\Omega}$ : $f$ equals the identity function on $\Omega$, which extends to a meromorphic function on the double $\widehat{\Omega}$ by means of the Schwarz function, and $f^{*}$ is the conjugate of the reflection of $f$ with respect to the involution on $\widehat{\Omega}$. In Section 8 we use formula (6) to construct explicit examples of classical quadrature domains.

In Section 6 we discuss the meromorphic resultant $\mathcal{R}(f, g)$ as a function of the quotient

$$
h(z)=\frac{f(z)}{g(z)} .
$$

Clearly, $f$ and $g$ are not uniquely determined by $h$ in this representation, but given $h$ it is easy to see that there are, up to constant factors, only finitely many pairs $(f, g)$ with non-zero resultant $\mathcal{R}(f, g)$ for which $(7)$ holds. Thus, the natural problem of characterizing the total range $\sigma(h)$ of these values $\mathcal{R}(f, g)$ arises.

Another case of interest is that the divisors of $f$ and $g$ are confined to lie in prescribed disjoint sets. This makes $\mathcal{R}(f, g)$ uniquely determined by $h$ and connects the subject to classical work of E. Bezout and L. Kronecker on representations of the classical resultant $\mathcal{R}_{\text {pol }}(f, g)$ by Toeplitz-structured determinants with entries equal to Laurent coefficients of the quotient $h(z)$. 
The 60's and 70's brought renewed interest to this area in connection with asymptotic behavior of truncated Toeplitz determinants for rational generating functions (cf. [3], [12], [15]). This problem naturally occurs in statistical mechanics in the study of the spinspin correlations for the twodimensional Ising model (see, e.g., [6]) and in quantum many body systems $[\mathbf{1 7}],[2]$.

One of the general results for rational symbols is an exact formula given by M. Day [12] in 1975. Suppose that $h$ is a rational function with simple zeros which is regular on the unit circle and does not vanish at the origin and infinity: $\operatorname{ord}_{0} h \leq 0, \operatorname{ord}_{\infty} h \leq 0$. Then for any $N \geq 1$ :

$$
\operatorname{det}\left(h_{i-j}\right)_{1 \leq i, j \leq N}=\sum_{i=1}^{p} r_{i} H_{i}^{N}, \quad h_{k}=\frac{1}{2 \pi} \int_{0}^{2 \pi} e^{-\mathrm{i} k \theta} h\left(e^{\mathrm{i} \theta}\right) d \theta,
$$

where $p, r_{i}, H_{i}$ are suitable rational expressions in the divisor of $h$. An accurate analysis of these expressions reveals the following interpretation of the above identity in terms of resultants:

$$
\frac{\operatorname{det}\left(h_{i-j}\right)_{1 \leq i, j \leq N}}{h^{N}(0)}=\sum \mathcal{R}\left(z^{N} f, g\right),
$$

where the (finite) sum is taken over all pairs $(f, g)$ satisfying $(7)$ such that $g$ is normalized by $g(\infty)=1$ and the divisor of zeros of $g$ coincides with the restriction of the polar divisor of $h$ to the unit disk: $(g)_{+}=(h)_{-} \cap \mathbb{D}$.

In the above notation, the equality (8) can be thought of as an identity between the elements of $\sigma(h)$ with a prescribed partitioning of the divisor. In Section 6.1 we consider resultant identities in the genus zero case in general, and show that there is a family of linear relations on $\sigma(h)$. These identities may be formally interpreted as a limiting case (for $N=0$ ) of the above Day formula (8). Moreover, our resultant identities are similar to those given recently by A. Lascoux and P. Pragacz [32] for Sylvester's double sums. On the other hand, by specializing the divisor $h$ we obtain a family of trigonometric identities generalizing known trigonometric addition theorems. Some of these identities were obtained recently by F. Calogero in $[\mathbf{7}, \mathbf{8}]$. For non-zero genus surfaces the situation with describing $\sigma(h)$ becomes much more complicated. We consider some examples for a complex torus, which indicates a general tight connection between resultant identities and addition theorems for theta-functions.

Returning to (6) and comparing this identity with determinantal representation (3) we find it reasonable to conjecture that one can associate to any compact Riemann surface an appropriate functional calculus for which the elimination function becomes a Fredholm determinant. In Section 7 we demonstrate such a model for the zero genus case. We show that the meromorphic resultant of two rational functions is given by a determinant of a multiplicative commutator of two Toeplitz operators on an appropriate Hardy space. There are interesting similarities between our determinantal representation (cf. formula (56) below) of the meromorphic resultant and the tau-function for solutions of some integrable hierarchies (see, for instance, $[43])$.

Further aspects of the meromorphic resultant discussed in the paper are interpretations in terms of potential theory, in Section 5, and various 
cohomological points of view, e.g., an expression of the resultant in terms of the Serre duality pairing (subsections 6.3 and 6.4). In Section 4 we give an independent proof of the symmetry of the resultant using the formalism of currents, and also derive several integral representations. Section 3 contains the main definitions and other preliminary material, and in Section 2 we review the polynomial resultant.

The authors are grateful to Mihai Putinar, Emma Previato and Yurii Neretin for many helpful comments and to the Swedish Research Council and the Swedish Royal Academy of Sciences for financial support. This research is a part of the European Science Foundation Networking Programme "Harmonic and Complex Analsyis and Applications HCAA".

\section{The polynomial resultant}

The resultant of two polynomials, $f$ and $g$, in one complex variable is a polynomial function in the coefficients of $f, g$ having the elimination property that it vanishes if and only if $f$ and $g$ have a common zero [54]. The resultant is a classical concept which goes back to the work of L. Euler, E. Bézout, J. Sylvester and A. Cayley. Traditionally, it plays an important role in algorithmic algebraic geometry as an effective tool for elimination of variables in polynomial equations. The renaissance of the classical theory of elimination in the last decade owes much to recent progress in toric geometry, complexity theory and the theory of univariate and multivariate residues of rational forms (see, for instance, $[\mathbf{1 9}],[\mathbf{4 9}],[\mathbf{5 2}],[\mathbf{1 0}]$ ).

We begin with some basic definitions and facts. In terms of the zeros of polynomials

$$
f(z)=f_{m} \prod_{i=1}^{m}\left(z-a_{i}\right)=\sum_{i=0}^{m} f_{i} z^{i}, \quad g(z)=g_{n} \prod_{j=1}^{n}\left(z-c_{j}\right)=\sum_{j=0}^{n} g_{j} z^{j},
$$

the resultant is given by the Poisson product formula $[\mathbf{1 9}]$

$$
\mathcal{R}_{\mathrm{pol}}(f, g)=f_{m}^{n} g_{n}^{m} \prod_{i, j}\left(a_{i}-c_{j}\right)=f_{m}^{n} \prod_{i=1}^{m} g\left(a_{i}\right)=(-1)^{m n} g_{n}^{m} \prod_{j=1}^{n} f\left(c_{j}\right) .
$$

It follows immediately from this definition that $\mathcal{R}_{\text {pol }}(f, g)$ is skew-symmetric and multiplicative:

$$
\mathcal{R}_{\text {pol }}(f, g)=(-1)^{m n} \mathcal{R}_{\text {pol }}(g, f), \quad \mathcal{R}_{\text {pol }}\left(f_{1} f_{2}, g\right)=\mathcal{R}_{\text {pol }}\left(f_{1}, g\right) \mathcal{R}_{\text {pol }}\left(f_{2}, g\right) .
$$

Alternatively, the resultant is uniquely (up to a normalization) defined as the irreducible integral polynomial in the coefficients of $f$ and $g$ which vanishes if and only if $f$ and $g$ have a common zero.

All known explicit representations of the polynomial resultant appear as certain determinants in the coefficients of the polynomials. Below we briefly comment on the most important determinantal representations. The interested reader may consult the recent monograph [19] and the surveys $[\mathbf{1 0}],[49]$, where further information on the subject can be found.

With $f, g$ as above, let us define an operator $S: \mathcal{P}_{n} \oplus \mathcal{P}_{m} \rightarrow \mathcal{P}_{m+n}$ by the rule:

$$
S(X, Y)=f X+g Y
$$


where $\mathcal{P}_{k}$ denotes the space of polynomials of degree $\leq k-1\left(\operatorname{dim} \mathcal{P}_{k}=k\right)$. Then

$$
\mathcal{R}_{\text {pol }}(f, g)=\operatorname{det}\left(\begin{array}{cccccc}
f_{0} & & & g_{0} & & \\
\vdots & \ddots & & \vdots & \ddots & \\
f_{m} & & f_{0} & g_{n} & & g_{0} \\
& \ddots & \vdots & & \ddots & \vdots \\
& & f_{m} & & & g_{n}
\end{array}\right)
$$

where the latter is the Sylvester matrix representing $S$ with respect to the monomial basis.

An alternative method to describe the resultant is the so-called BézoutCayley formula. For $\operatorname{deg} f=\operatorname{deg} g=n$ it reads

$$
\mathcal{R}_{\text {pol }}(f, g)=\operatorname{det}\left(\beta_{i j}\right)_{0 \leq i, j \leq n-1},
$$

where

$$
\frac{f(z) g(w)-f(w) g(z)}{z-w}=\sum_{i, j=0}^{n-1} \beta_{i j} z^{i} w^{j},
$$

is the Bézoutian of $f$ and $g$. The general case, say $\operatorname{deg} f<\operatorname{deg} g$, is obtained from (11) and (13) by completing $f(z)$ to $z^{k} g(z), k=\operatorname{deg} g-\operatorname{deg} f$.

Other remarkable representations of the resultant are given as determinants of Toeplitz-structured matrices with entries equal to Laurent coefficients of the quotient $h(z)=\frac{f(z)}{g(z)}$. These formulas were known already to E. Bezout and were rediscovered and essentially developed later by J. Sylvester and L. Kronecker in connection to finding of the greatest common divisor of two polynomials (see Chapter 12 in $[\mathbf{1 9}]$ and $[\mathbf{4}]$ ).

Recently, a similar formula in terms of contour integrals of the quotient $h(z)$ has been given by R. Hartwig [28] (see also M. Fisher and R. Hartwig [15]). In its simplest form this formula reads as follows. With $f$ and $g$ as in (9), we assume $g_{0}=g(0) \neq 0$. Then for any $N \geq n$, the polynomial resultant, up to a constant factor, is the truncated Toeplitz determinant for the symbol $h(z)$ :

$$
\mathcal{R}_{\mathrm{pol}}(f, g)=f_{m}^{n-N} g_{0}^{m+N} \operatorname{det} t_{m, N}(h),
$$

where $h(z)=\sum_{k=0}^{\infty} h_{k} z^{k}$ is the Taylor development of the quotient around $z=0$ and

$$
t_{m, N}(h)=\left(\begin{array}{cccc}
h_{m} & h_{m-1} & \ldots & h_{m-N+1} \\
h_{m+1} & h_{m} & \ldots & h_{m-N+2} \\
\vdots & \vdots & \ddots & \vdots \\
h_{m+N-1} & h_{m+N-2} & \ldots & h_{m}
\end{array}\right),
$$

and $h_{k}=0$ for negative $k$.

The determinant $\operatorname{det} t_{m, N}(h)$ is a commonly used object in theory of Toeplitz operators. For instance, the celebrated Szegö limit theorem (see, e.g., [6]) states that, under some natural assumptions, $\operatorname{det} t_{0, N}(h)$ behaves like a geometric progression. Exact formulations will be given in Section 7.1, where the above identity is generalized to the meromorphic case.

It is worth mentioning here another powerful and rather unexpected application of $\operatorname{det} t_{m, N}(h)$, the so-called Thom-Porteous formula in the theory 
of determinantal varieties $[\mathbf{1 8}],[\mathbf{2 0}$, p. 415]. We briefly describe this identity in the classical setup. Consider an $n \times m(n \leq m)$ matrix $A$ with entries $a_{i j}$ being homogeneous forms in the variables $x_{1}, \ldots, x_{k}$ of degree $p_{i}+q_{j}$ (for some integers $\left.p_{i}, q_{j}\right)$. Denote by $V_{r}$ the locus of points in $\mathbb{P}^{k}$ at which the rank of $A$ is at most $r$. Then, thinking of $p_{i}, q_{j}$ as formal variables, one has

$$
\operatorname{deg} V_{r}=\operatorname{det} t_{m-r, n-r}(c), \quad \sum_{k=0}^{\infty} c_{k} z^{k}=\frac{\prod_{j=1}^{m}\left(1+q_{j} z\right)}{\prod_{i=1}^{n}\left(1-p_{i} z\right)} .
$$

We mention here also a differential analog of the polynomial resultant in algebraic theory of commuting (linear) ordinary differential operators. A key observation goes back to J.L. Burchnall and T.W. Chaundy and states that commuting ordinary differential operators satisfy an equation for a certain algebraic curve, the so-called spectral curve of the corresponding operators (see $[\mathbf{3 6}]$ for a detailed discussion and historical remarks). The defining equation of the curve is equivalent to the vanishing of a determinant of a Sylvester-type matrix. This phenomenon was a main ingredient of the modern fundamental algebro-geometric approach initiated by I. Krichever [31] in the theory of integrable equations. By using the Burchnall-ChaundyKrichever correspondence between meromorphic functions on a suitable Riemann surface and differential operators, E. Previato in [35] succeeded to get a pure algebraic version of the proof of Weil's reciprocity.

All the determinantal formulas given above fit into a general scheme: given a pair of polynomials one can associate an operator $S$ in a suitable coefficient model space such that $\mathcal{R}_{\text {pol }}(f, g)=\operatorname{det} S$. On the other hand, none of the models behaves well under multiplication of polynomials. This makes it difficult to translate identities like (11) into matrix language. One way to get around this difficulty is to observe that (14) is a special case of the Szegö strong limit theorem for rational symbols [15] and to consider infinite dimensional determinantal (Fredholm) models instead. We sketch such a model in Section 7 below.

\section{The meromorphic resultant}

3.1. Preliminary remarks. For rational functions with neither zeros nor poles at infinity, say

$$
f(z)=\lambda \prod_{i=1}^{m} \frac{z-a_{i}}{z-b_{i}}, \quad g(z)=\mu \prod_{j=1}^{n} \frac{z-c_{j}}{z-d_{j}},
$$

$\left(\lambda, \mu \neq 0\right.$ and all $a_{i}, b_{i}, c_{j}, d_{j}$ distinct $)$ it is natural to define the resultant as

$$
\mathcal{R}(f, g)=\prod_{i=1}^{m} \frac{g\left(a_{i}\right)}{g\left(b_{i}\right)}=\prod_{j=1}^{n} \frac{f\left(c_{j}\right)}{f\left(d_{j}\right)} .
$$

In other words,

$$
\mathcal{R}(f, g)=\prod_{i=1}^{m} \prod_{j=1}^{n} \frac{a_{i}-c_{j}}{a_{i}-d_{j}} \cdot \frac{b_{i}-d_{j}}{b_{i}-c_{j}}=\prod_{i=1}^{m} \prod_{j=1}^{n}\left(a_{i}, b_{i}, c_{j}, d_{j}\right),
$$

where $(a, b, c, d):=\frac{a-c}{a-d} \cdot \frac{b-d}{b-c}$ is the classical cross ratio of four points. 
Note that (nonconstant) polynomials do not fit into this picture since they always have a pole at infinity, but the polynomial resultant can still be recovered by a localization procedure (see Section 9). Notice also that the above resultant for rational functions actually has better properties than the polynomial resultant, e.g., it is symmetric $(\mathcal{R}(f, g)=\mathcal{R}(g, f))$, homogenous of degree zero and it only depends on the divisors of $f$ and $g$. The resultant for meromorphic functions on a compact Riemann surface will be modeled on the above definition (16) and contain it as a special case.

3.2. Divisors and their actions. We start with a brief discussion of divisors. A divisor on a Riemann surface $M$ is a finite formal linear combination of points on $M$, i.e., an expression of the form

$$
D=\sum_{i=1}^{m} n_{i} a_{i}
$$

$a_{i} \in M, n_{i} \in \mathbb{Z}$. Thus a divisor is the same thing as a 0 -chain, which acts on 0 -forms, i.e., functions, by integration. Namely, the divisor (18) acts on functions $\varphi$ by

$$
\langle D, \varphi\rangle=\int_{D} \varphi=\sum_{i=1}^{m} n_{i} \varphi\left(a_{i}\right) .
$$

From another (dual) point of view divisors can be looked upon as maps $M \rightarrow \mathbb{Z}$ with support at a finite number of points, namely the maps which evaluate the coefficients in expressions like (18). If $D$ is a divisor as in (18) we also write $D: M \rightarrow \mathbb{Z}$ for the corresponding evaluation map. Then $D=\sum_{a \in M} D(a) a$. The degree of $D$ is

$$
\operatorname{deg} D=\sum_{i=1}^{m} n_{i}=\sum_{a \in M} D(a) .
$$

and its support is

$$
\operatorname{supp} D=\{a \in M: D(a) \neq 0\} .
$$

If $f: M \rightarrow \mathbb{P}$ is a nonconstant meromorphic function and $\alpha \in \mathbb{P}$ then the inverse image $f^{-1}(\alpha)$, with multiplicities counted, can be considered as a (positive) divisor in a natural way. The divisor of $f$ then is

$$
(f)=f^{-1}(0)-f^{-1}(\infty) .
$$

If $f$ is constant, not 0 or $\infty$, then $(f)=0$ (the zero element in the Abelian group of divisors).

Recall that any divisor of the form (20) is called a principal divisor. In the dual picture the same divisor acts on points as follows:

$$
(f)(a)=\operatorname{ord}_{a}(f),
$$

where $\operatorname{ord}_{a}(f)$ is the integer $m$ such that, in terms of a local coordinate $z$,

$$
f(z)=c_{m}(z-a)^{m}+c_{m+1}(z-a)^{m+1}+\ldots \text { with } \quad c_{m} \neq 0 .
$$

By ord $f$ we denote the order of $f$, that is the cardinality of $f^{-1}(0)$.

Divisors act on functions by (19). We can also let functions act on divisors. In this case we shall, by convention, let the action be multiplicative 
rather than additive: if $h=h\left(u_{1}, \ldots, u_{k}\right)$ is a function and $D_{1}, \ldots, D_{k}$ are divisors, we set

$$
h\left(D_{1}, \ldots, D_{k}\right)=\prod_{a_{1}, \ldots, a_{k} \in M} h\left(a_{1}, \ldots, a_{k}\right)^{D_{1}\left(a_{1}\right) \cdots D_{k}\left(a_{k}\right)},
$$

whenever this is well-defined. Observe that this definition is consistent with the standard evaluation of a function at a point. Indeed, any point $a \in M$ may be regarded simultaneously as a divisor $D_{a}=a$. Then $h\left(a_{1}, \ldots, a_{p}\right)=$ $h\left(D_{a_{1}}, \ldots, D_{a_{p}}\right)$. In what follows we make no distinction between $D_{a}$ and $a$.

With branches of the logarithm chosen arbitrarily (21) can also be written

$$
h\left(D_{1}, \ldots, D_{p}\right)=\exp \left\langle D_{1} \otimes \ldots \otimes D_{p}, \log h\right\rangle .
$$

When $D_{i}, i=1, \ldots, p$ are principal divisors, say $D_{i}=\left(g_{i}\right)$ for some meromorphic functions $g_{i}$, the definition (21) yields

$$
h\left(\left(g_{1}\right), \ldots,\left(g_{p}\right)\right)=\prod_{a_{1}, \ldots, a_{p} \in M} h\left(a_{1}, \ldots, a_{p}\right)^{\operatorname{ord}_{a_{1}}\left(g_{1}\right) \cdots \operatorname{ord}_{a_{p}}\left(g_{p}\right)} .
$$

3.3. Main definitions. Let now $f, g$ be meromorphic functions (not identically 0 and $\infty$ ) on an arbitrary compact Riemann surface $M$ and let their divisors be

$$
\begin{aligned}
& (f)=f^{-1}(0)-f^{-1}(\infty)=\sum_{i=1}^{m} a_{i}-\sum_{i=1}^{m} b_{i}, \\
& (g)=g^{-1}(0)-g^{-1}(\infty)=\sum_{j=1}^{n} c_{j}-\sum_{j=1}^{n} d_{j} .
\end{aligned}
$$

At first we assume that $(f)$ and $(g)$ are "generic" in the sense of having disjoint supports. In view of the suggested resultant (16) for rational functions the following definition is natural.

Definition 1. The (meromorphic) resultant of two generic meromorphic functions $f$ and $g$ as above is

$$
\mathcal{R}(f, g)=g((f))=\prod_{i=1}^{m} \frac{g\left(a_{i}\right)}{g\left(b_{i}\right)}=\frac{g\left(f^{-1}(0)\right)}{g\left(f^{-1}(\infty)\right)}=\exp \langle(f), \log g\rangle .
$$

In the last expression, an arbitrary branch of $\log g$ can be chosen at each point of $(f)$.

Elementary properties of the resultant are multiplicativity in each variable:

$$
\mathcal{R}\left(f_{1} f_{2}, g\right)=\mathcal{R}\left(f_{1}, g\right) \mathcal{R}\left(f_{2}, g\right), \quad \mathcal{R}\left(f, g_{1} g_{2}\right)=\mathcal{R}\left(f, g_{1}\right) \mathcal{R}\left(f, g_{2}\right) .
$$

An important observation is homogeneity of degree zero

$$
\mathcal{R}(a f, b g)=\mathcal{R}(f, g)
$$

for $a, b \in \mathbb{C}^{*}:=\mathbb{C} \backslash\{0\}$. The latter implies that $\mathcal{R}(f, g)$ depends merely on the divisors $(f)$ and $(g)$.

Less elementary, but still true, is the symmetry:

$$
\mathcal{R}(f, g)=\mathcal{R}(g, f)
$$


i.e., in the terms of the divisors

$$
\prod_{i} \frac{g\left(a_{i}\right)}{g\left(b_{i}\right)}=\prod_{j} \frac{f\left(c_{j}\right)}{f\left(d_{j}\right)} .
$$

This is a consequence of Weil's reciprocity law [55], [20, p. 242]. In Section 4 we shall find some integral formulas for the resultant and also give an independent proof of (25).

If, in (21), some of the divisors $D_{k}$ are principal then the resulting action $h$ may be written as a composition of the corresponding resultants. For instance, for a function $h$ of two variables we have

$$
h((f),(g))=\mathcal{R}_{u}\left(f(u), \mathcal{R}_{v}(g(v), h(u, v))\right),
$$

where $\mathcal{R}_{u}$ denotes the resultant in the $u$-variable.

REMARK 1. The definition of meromorphic resultant naturally extends to more general objects than meromorphic functions. Indeed, of $f$ we need only its divisor and $g$ may be a fairly arbitrary function. We shall still use (23) as a definition in such extended contexts. However, there is no symmetry relation like (25) in general. See e.g. Lemma 4.

When, as above, $(f)$ and $(g)$ have disjoint supports $\mathcal{R}(f, g)$ is a nonzero complex number. It is important to extend the definition of $\mathcal{R}(f, g)$ to certain cases when $(f)$ and $(g)$ do have common points.

Definition 2. A pair of two meromorphic functions $f$ and $g$ is said to be admissible on a set $A \subset M$ if the function $a \rightarrow \operatorname{ord}_{a}(g) \operatorname{ord}_{a}(f)$ is sign semi-definite on $A$ (i.e., is either $\geq 0$ on all $A$ or $\leq 0$ on all $A$ ). If $A=M$ we shall simply say that $f$ and $g$ is an admissible pair.

It is easily seen that the product in (23) is well-defined as a complex number or $\infty$ whenever $f$ and $g$ form an admissible pair.

Clearly, any pair of two meromorphic functions whose divisors have no common points is admissible (we call such pairs generic). Another important example is the family of all polynomials, regarded as meromorphic functions on the Riemann sphere $\mathbb{P}$. It is easily seen that any pair of polynomials is admissible with respect to an arbitrary subset $A \subset \mathbb{P}$.

The following elimination property is an immediate corollary of the definitions.

Proposition 1. Let two nonconstant meromorphic functions $f, g$ form an admissible pair on $M$. Then $\mathcal{R}(f, g)=0$ if and only if $f$ and $g$ have a common zero or a common pole. In particular, $\mathcal{R}(f, g)=0$ if $f$ and $g$ are polynomials.

3.4. Elimination function. We have seen above that the meromorphic resultant of two individual functions is not always well-defined (namely, if the two functions do not form an admissible pair). However one may still get useful information by embedding the functions in families depending on parameters, for example by taking the resultant of $f-z$ and $g-w$. We shall see in Section 8.3 that such resolved versions of the resultant have additional analytic advantages. 
Let $z, w \in \mathbb{C}$ be free variables. The expression

$$
\mathcal{E}(z, w) \equiv \mathcal{E}_{f, g}(z, w)=\mathcal{R}(f-z, g-w),
$$

if defined, will be called the elimination function of $f$ and $g$.

THEOREM 1. Let $f$ and $g$ be nonconstant meromorphic functions without common poles. Then the elimination function is well defined everywhere except for finitely many pairs $(z, w)$, and it is a rational function of the form

$$
\mathcal{E}(z, w)=\frac{Q(z, w)}{P(z) R(w)}
$$

where $Q, P, R$ are polynomials, and

$$
P(z)=\prod_{d \in g^{-1}(\infty)}(z-f(d)), \quad R(w)=\prod_{b \in f^{-1}(\infty)}(w-g(b)) .
$$

Proof. Note that a linear transformation $f \rightarrow f-z$ keeps the polar locus unchanged. Thus the elimination function $\mathcal{R}(f-z, g-w)$ is welldefined for all pairs $(z, w)$ such that $f^{-1}(z) \cap g^{-1}(\infty)=g^{-1}(w) \cap f^{-1}(\infty)=\emptyset$. Let $(z, w)$ be any such pair. Then applying the symmetry relation (25) we obtain

$$
\mathcal{E}(z, w)=\frac{(g-w)\left(f^{-1}(z)\right)}{(g-w)\left(f^{-1}(\infty)\right)}=\frac{(f-z)\left(g^{-1}(w)\right)}{(f-z)\left(g^{-1}(\infty)\right)} .
$$

Let $f, g$ have orders $m$ and $n$, respectively, as in (22), and let $\left\{f_{i}^{-1}\right\}$ denote the branches of $f^{-1}$. Then spelling out the meaning we find, using that the symmetric functions of $\left\{g\left(f_{i}^{-1}(z)\right)\right\}$ are single-valued from the Riemann sphere into itself, hence are rational functions, that

$(g-w)\left(f^{-1}(z)\right)=\prod_{i=1}^{m}\left(g\left(f_{i}^{-1}(z)\right)-w\right)=(-1)^{m}\left(w^{m}+R_{1}(z) w^{m-1}+\cdots+R_{m}(z)\right)$,

where the $R_{i}(z)$ are rational. Similarly,

$$
(g-w)\left(f^{-1}(\infty)\right)=(-1)^{m}\left(w^{m}+r_{1} w^{m-1}+\cdots+r_{m}\right),
$$

where the $r_{i}$ are constants.

With the same kind of arguments for $(f-z)\left(g^{-1}(w)\right)$ and $(f-z)\left(g^{-1}(\infty)\right)$ we obtain

$\mathcal{E}(z, w)=\frac{w^{m}+R_{1}(z) w^{m-1}+\cdots+R_{m}(z)}{w^{m}+r_{1} w^{m-1}+\cdots+r_{m}}=\frac{z^{n}+P_{1}(w) z^{n-1}+\cdots+P_{n}(w)}{z^{n}+p_{1} z^{n-1}+\cdots+p_{n}}$.

Clearing the denominators (in the numerators) yields the required statement.

Important, and useful in applications, is the following elimination property of the function $\mathcal{E}_{f, g}(z, w)$. Let us choose $\zeta \in M$ arbitrarily and insert $z=f(\zeta), w=g(\zeta)$ into $\mathcal{E}_{f, g}(z, w)$. Since the functions $f-z$ and $g-w$ then have a common zero (namely at $\zeta$ ) this gives, by Proposition 1, that

$$
\mathcal{E}_{f, g}(f(\zeta), g(\zeta))=0 \quad(\zeta \in M) .
$$

In particular,

$$
Q(f, g)=0,
$$

i.e., we have recovered the classical polynomial relation between two functions on a compact Riemann surface (see [14], [16], for example). 
3.5. Extended elimination function. We have seen that the elimination function is well-defined for any pair of meromorphic functions without common poles. One step further, linear fractional transformations allow us to refine the definition of elimination function in such a way that it becomes well-defined for all pairs of meromorphic functions.

Namely, let $f$ and $g$ be two arbitrary meromorphic functions and consider the function of four complex variables:

$$
\mathcal{E}\left(z, w ; z_{0}, w_{0}\right) \equiv \mathcal{E}_{f, g}\left(z, w ; z_{0}, w_{0}\right)=\mathcal{R}\left(\frac{f-z}{f-z_{0}}, \frac{g-w}{g-w_{0}}\right) .
$$

Let us choose arbitrary the pair $\left(z, z_{0}\right)$. Then we have for divisor: $\left(\frac{f-z}{f-z_{0}}\right)=f^{-1}(z)-f^{-1}\left(z_{0}\right)$. It is easy to see that the resultant in (27) is well defined for any quadruple $\left(z, w ; z_{0}, w_{0}\right)$ with

$$
\left[g^{-1}(w) \cup g^{-1}\left(w_{0}\right)\right] \cap\left[f^{-1}(z) \cup f^{-1}\left(z_{0}\right)\right]=\emptyset .
$$

The set $X$ of all $\left(z, w ; z_{0}, w_{0}\right)$ such that $(28)$ holds is a dense open subset of in $\mathbb{C}^{4}$.

Applying then an argument similar to that in Theorem 1, we find that the right hand side in $(27)$ is a rational function for $\left(z, w ; z_{0}, w_{0}\right) \in X$. We call this function the extended elimination function of $f$ and $g$.

We have the cross-ratio-like symmetries $\mathcal{E}\left(z, w ; z_{0}, w_{0}\right)=\mathcal{E}\left(z_{0}, w_{0} ; z, w\right)$, and

$$
\mathcal{E}\left(z, w_{0} ; z_{0}, w\right)=\frac{1}{\mathcal{E}\left(z, w ; z_{0}, w_{0}\right)} .
$$

In the case when the elimination function $\mathcal{E}_{f, g}(z, w)$ is well-defined we have the following reduction:

$$
\mathcal{E}\left(z, w ; z_{0}, w_{0}\right)=\frac{\mathcal{E}(z, w) \mathcal{E}\left(z_{0}, w_{0}\right)}{\mathcal{E}\left(z, w_{0}\right) \mathcal{E}\left(z_{0}, w\right)}=\frac{Q(z, w) Q\left(z_{0}, w_{0}\right)}{Q\left(z, w_{0}\right) Q\left(z_{0}, w\right)}
$$

with $Q$ as in Theorem 1 .

In the other direction, the ordinary elimination function, if well-defined, can be viewed as a limiting case of the extended version. Indeed, it follows from null-homogeneity of the meromorphic resultant that

$$
\mathcal{E}\left(z, w ; z_{0}, w_{0}\right)=\mathcal{R}\left(\frac{f-z}{1-f / z_{0}}, \frac{g-w}{1-g / w_{0}}\right),
$$

and therefore that

$$
\lim _{z_{0}, w_{0} \rightarrow \infty} \mathcal{E}\left(z, w ; z_{0}, w_{0}\right)=\mathcal{E}(z, w) .
$$

There are still cases when the elimination function is not defined or is trivial while its extended version contains information. To illustrate this, let us consider a meromorphic function $f$ of order $n$ and let $g=f$. Then a straightforward computation reveals that

$$
\mathcal{E}_{f, f}\left(z, w ; z_{0}, w_{0}\right)=\left(\frac{z-z_{0}}{z-w_{0}} \cdot \frac{w-w_{0}}{w-z_{0}}\right)^{n}=\left(z, w, z_{0}, w_{0}\right)^{n}
$$

where $\left(z, w, z_{0}, w_{0}\right)$ is the cross ratio. 
3.6. The meromorphic resultant on surfaces with small genera. On the Riemann sphere $\mathbb{P}$ the resultant reduces to a product of cross ratios (17) and the symmetry relation (25) becomes trivial. Note that the cross ratio itself may be regarded as the meromorphic resultant of two linear fractional functions.

From a computational point of view, evaluation of the meromorphic resultant on $\mathbb{P}$ is similar to the evaluation of polynomial resultants. Indeed, for any admissible rational functions given by the ratio of polynomials, $f=$ $f_{1} / f_{2}$ and $g=g_{1} / g_{2}$, one finds that

$$
\mathcal{R}(f, g)=f(\infty)^{\operatorname{ord}_{\infty}(g)} g(\infty)^{\operatorname{ord}_{\infty}(f)} \cdot \frac{\mathcal{R}_{\mathrm{pol}}\left(f_{1}, g_{1}\right) \mathcal{R}_{\mathrm{pol}}\left(f_{2}, g_{2}\right)}{\mathcal{R}_{\mathrm{pol}}\left(f_{1}, g_{2}\right) \mathcal{R}_{\mathrm{pol}}\left(f_{2}, g_{1}\right)}
$$

The latter formula combined with formulas in Section 2 expresses the meromorphic resultant in terms of the coefficients of the representing polynomials of $f$ and $g$. For example, since each resultant in (29) is a Sylvester determinant (12),

$$
\mathcal{R}_{\text {pol }}\left(f_{i}, g_{j}\right)=\operatorname{det} S\left(f_{i}, g_{j}\right) \equiv \operatorname{det} S_{i j},
$$

the resulting product amounts to

$$
\mathcal{R}(f, g)=f(\infty)^{\operatorname{ord}_{\infty}(g)} g(\infty)^{\operatorname{ord}_{\infty}(f)} \cdot \operatorname{det}\left(S_{12}^{-1} S_{11} S_{21}^{-1} S_{22}\right) .
$$

In Section 7 we give another, more invariant, approach to the representation of meromorphic resultants via determinants (see also Section 7.2 for the exponential representations of $\mathcal{R}(f, g)$ ).

Now we spell out the definition of the resultant in case of Riemann surfaces of genus one. Consider the complex torus $M=\mathbb{C} / L_{\tau}$, where $L_{\tau}=$ $\mathbb{Z}+\tau \mathbb{Z}$ is the lattice formed by $\tau \in \mathbb{C}, \operatorname{Im} \tau>0$. A meromorphic function on $M$ is represented as an $L_{\tau}$-periodic function on $\mathbb{C}$. Let

$$
\theta(\zeta)=\theta_{11}(\zeta) \equiv \sum_{k=-\infty}^{\infty} e^{\pi \mathrm{i}\left(k^{2} \tau+k(1+\tau+2 \zeta)\right)}
$$

be the Jacobi theta-function. Then any meromorphic function $f$ on $M$ is given by a ratio of translated theta-functions:

$$
f(\zeta)=\lambda \prod_{i=1}^{m} \frac{\theta\left(\zeta-a_{i}\right)}{\theta\left(\zeta-b_{i}\right)},
$$

and a necessary and sufficient condition that such a ratio really defines a meromorphic function is that the divisor is principal, i.e., by Abel's theorem, that

$$
\sum_{i=1}^{m}\left(a_{i}-b_{i}\right) \in L .
$$

With $f$ as above and $g$ similarly with $c_{j}$ and $d_{j}, \sum_{j=1}^{n}\left(c_{j}-d_{j}\right) \in L$, the following representation for the meromorphic resultant on the torus holds:

$$
\mathcal{R}(f, g)=\prod_{i=1}^{m} \prod_{j=1}^{n} \frac{\theta\left(c_{j}-a_{i}\right) \theta\left(d_{j}-b_{i}\right)}{\theta\left(c_{j}-b_{i}\right) \theta\left(d_{j}-a_{i}\right)} .
$$




\section{Integral representations}

4.1. Integral formulas. We shall derive some integral representations for the meromorphic resultant, and in passing also give a proof of the symmetry (25), Weil's reciprocity law. Let $f, g$ be nonconstant meromorphic functions on a compact Riemann surface $M$ of genus $p \geq 0$ and recall (23) that the resultant can be written

$$
\mathcal{R}(f, g)=\exp \langle(f), \log g\rangle .
$$

We assume that the divisors $(f)$ and $(g)$ have disjoint supports. Since $(f)$ is integer-valued and different branches of $\log g$ differ by integer multiples of $2 \pi \mathrm{i}$ it does not matter which branch of $\log g$ is chosen at each point of $(f)$. However, our present aim is to treat $\log g$ as a global object on $M$, in order to interpret $\langle(f), \log g\rangle$ as a current acting on a function and to write it as an integral over $M$.

First of all, to any divisor $D$ can be naturally associated a 2 -form current $\mu_{D}$ (a 2-form with distribution coefficients), which represents $D$ in the sense that

$$
\langle D, \varphi\rangle=\int_{D} \varphi=\int_{M} \varphi \wedge \mu_{D}
$$

for smooth functions $\varphi$. With $D=\sum n_{i} a_{i}$ this $\mu_{D}$ is of course just

$$
\mu_{D}=\delta_{D} d x \wedge d y=\sum n_{i} \delta_{a_{i}} d x \wedge d y,
$$

where $\delta_{a}$ is the Dirac delta at the point $a$ and with respect to a local variable $z=x+\mathrm{i} y$ chosen (only $\delta_{a} d x \wedge d y$ has an invariant meaning). When $D=(f)$ we have the following formula.

LEMMA 1. If $f$ is a meromorphic function, then $\mu_{(f)}=\frac{1}{2 \pi \mathrm{i}} d\left(\frac{d f}{f}\right)$ in the sense of currents.

Proof. In a neighbourhood of a point $a$ with $\operatorname{ord}_{a}(f)=m$, i.e.,

$$
f(z)=c_{m}(z-a)^{m}+c_{m+1}(z-a)^{m+1}+\ldots, \quad c_{m} \neq 0,
$$

in terms of a local coordinate, we have $\frac{d f}{f}=\left(\frac{m}{z-a}+h(z)\right) d z$ with $h$ holomorphic. Hence,

$$
d\left(\frac{d f}{f}\right)=\frac{\partial}{\partial \bar{z}}\left(\frac{m}{z-a}+h(z)\right) d \bar{z} \wedge d z=m \pi \delta_{a} d \bar{z} \wedge d z=2 \pi \mathrm{i} m \delta_{a} d x \wedge d y,
$$

from which the lemma follows.

Next we shall make $\log f$ and $\log g$ single-valued on $M$ by making "cuts". Let $\alpha_{1}, \ldots, \alpha_{p}, \beta_{1}, \ldots, \beta_{p}$ be a canonical homology basis for $M$ such that each $\beta_{k}$ intersects $\alpha_{k}$ once from the right to the left $(k=1, \ldots, p)$ and no other crossings occur. We may choose these curves so that they do not meet the divisors $(f)$ and $(g)$.

Since the divisors $(f)$ and $(g)$ have degree zero we can write

$$
(f)=\partial \gamma_{f}, \quad(g)=\partial \gamma_{g}
$$

where $\gamma_{f}, \gamma_{g}$ are 1-chains. We may arrange these curves so that there are no intersections and so that they are contained in $M \backslash\left(\alpha_{1} \cup \cdots \cup \beta_{p}\right)$. 
Now, it is possible to select single-valued branches of $\log f$ and $\log g$ in

$$
M^{\prime}=M \backslash\left(\gamma_{f} \cup \gamma_{g} \cup \alpha_{1} \cup \cdots \cup \beta_{p}\right) .
$$

Fix such branches and denote them $\log f, \log g$. Then $\log f$ and $\log g$ are functions, defined almost everywhere on $M$, and $\log g$ is smooth in a neighbourhood of the support of $(f)$ and vice versa. In particular, $\langle(f), \log g\rangle$ and $\langle(g), \log f\rangle$ make sense.

Now using Lemma 1 and partial integration (with exterior derivatives taken in the sense of currents) we get

$$
\begin{aligned}
\mathcal{R}(f, g) & =\exp \langle(f), \log g\rangle=\exp \left[\int_{M} \mu_{(f)} \wedge \log g\right] \\
& =\exp \left[\frac{1}{2 \pi \mathrm{i}} \int_{M} d\left(\frac{d f}{f}\right) \wedge \log g\right]=\exp \left[\frac{1}{2 \pi \mathrm{i}} \int_{M} \frac{d f}{f} \wedge d \log g\right] .
\end{aligned}
$$

In summary:

TheOREM 2. Let $f$ and $g$ be two meromorphic functions on a compact Riemann surface whose divisors have disjoint supports. Then

$$
\mathcal{R}(f, g)=\exp \left[\frac{1}{2 \pi \mathrm{i}} \int_{M} \frac{d f}{f} \wedge d \log g\right] .
$$

In particular, for generic $z, w$,

$$
\mathcal{E}_{f, g}(z, w)=\exp \left[\frac{1}{2 \pi \mathrm{i}} \int_{M} \frac{d f}{f-z} \wedge d \log (g-w)\right] .
$$

It should be noted that the only contributions to the integrals above come from the jumps of $\log g$ (and $\log (g-w)$ respectively), because outside this set of discontinuities the integrand contains $d z \wedge d z=0$ as a factor.

4.2. Symmetry of the resultant. We proceed to study $d \log$ in detail. Let first $a, b$ be two points in the complex plane and $\gamma$ a curve from $b$ to $a$ such that $\partial \gamma=a-b$ (formal difference). Then, with a single-valued branch of the logarithm chosen in $\mathbb{C} \backslash \gamma$,

$$
\begin{aligned}
d \log \frac{z-a}{z-b} & =\frac{d z}{z-a}-\frac{d z}{z-b}+\mathrm{i}\left[d \operatorname{Arg} \frac{z-a}{z-b}\right]_{\text {jump contribution from } \gamma} \\
& =\frac{d z}{z-a}-\frac{d z}{z-b}-2 \pi \mathrm{i} d H_{\gamma}(z) .
\end{aligned}
$$

Here $d H_{\gamma}$ is the 1-form current supported by $\gamma$ and defined as the (distributional) differential of the function $H_{\gamma}$ which in a neighbourhood of any interior point of $\gamma$ equals +1 to the right of $\gamma$ and zero to the left. Thus $d H_{\gamma}$ is locally exact away from the end points. The function $H_{\gamma}$ cannot be defined in any full neighbourhood of $a$ or $b$. On the other hand, $d H_{\gamma}$ is taken to have no distributional contributions at $a$ and $b$. One easily checks that this gives a current which represents $\gamma$ in the sense that

$$
\int_{\gamma} \tau=\int_{M} d H_{\gamma} \wedge \tau
$$

for all smooth 1-forms $\tau$. Taking $\tau$ of the form $d \varphi$ gives

$$
\int_{M} d\left(d H_{\gamma}\right) \wedge \varphi=\int_{M} d H_{\gamma} \wedge d \varphi=\int_{\gamma} d \varphi=\int_{\partial \gamma} \varphi
$$


Thus the 0 -chain, or divisor, $\partial \gamma$ is represented by $d\left(d H_{\gamma}\right)$. We can write this also as $d\left(d H_{\gamma}\right)=\mu_{\partial \gamma}$, where $\mu_{D}$ is defined in (31). Note in particular that $d H_{\gamma}$ is not closed, despite the notation.

If $\gamma$ and $\sigma$ are two curves (1-chains) which cross each other at a point $c$, then it is easy to check (and well-known) that

$$
d H_{\gamma} \wedge d H_{\sigma}= \pm \delta_{c} d x \wedge d y
$$

with the plus sign if $\sigma$ crosses $\gamma$ from the right (of $\gamma$ ) to the left, the minus sign in the opposite case. For the curves $\alpha_{1}, \ldots, \beta_{p}$ in the canonical homology basis, the forms $d H_{\alpha_{1}}, \ldots, d H_{\beta_{p}}$ are closed, since the curves are themselves closed.

Now we extend the above analysis to $\log f$ in place of $\log \frac{z-a}{z-b}$. In addition to the jump across $\gamma_{f}$ (an arbitrary 1-chain in $M \backslash\left(\alpha_{1} \cup \ldots \cup \beta_{p}\right.$ ) with $\left.\partial \gamma_{f}=(f)\right)$ we need to take into account possible jumps across the $\alpha_{k}, \beta_{k}$. In order to reach the right hand side of $\alpha_{k}$ from the left hand side within $M^{\prime}$ one just follows $\beta_{k}$. The increase of $\log f$ along this curve is $\int_{\beta_{k}} \frac{d f}{f}$, hence this is also the jump of $\log f$ across $\alpha_{k}$, from the left to the right. With a similar analysis for the jump across $\beta_{k}$ one arrives at the following expression for $d \log f$ :

$$
d \log f=\frac{d f}{f}-2 \pi \mathrm{i}\left(d H_{\gamma_{f}}+\sum_{k=1}^{p}\left(\frac{1}{2 \pi \mathrm{i}} \int_{\beta_{k}} \frac{d f}{f} \cdot d H_{\alpha_{k}}-\frac{1}{2 \pi \mathrm{i}} \int_{\alpha_{k}} \frac{d f}{f} \cdot d H_{\beta_{k}}\right)\right) .
$$

This means that $\gamma_{f}$ needs to be modified to the 1-chain

$$
\sigma_{f}=\gamma_{f}+\sum_{k=1}^{p}\left(\operatorname{wind}_{\beta_{k}}(f) \cdot \alpha_{k}-\operatorname{wind}_{\alpha_{k}}(f) \cdot \beta_{k}\right),
$$

where, for a closed curve $\alpha$ in general, wind $\alpha(f)$ stands for the winding number

$$
\operatorname{wind}_{\alpha}(f)=\frac{1}{2 \pi \mathrm{i}} \int_{\alpha} \frac{d f}{f} \in \mathbb{Z} .
$$

Notice that $\partial \sigma_{f}=\partial \gamma_{f}=(f)$ and that now $\log f$ can be taken to be singlevalued analytic in $M \backslash \operatorname{supp} \sigma_{f}$. The above can be we can summarized as follows.

Lemma 2. Given any meromorphic function $f$ in $M$ there exists a 1chain $\sigma_{f}$ having the property that $\partial \sigma_{f}=(f), \log f$ has a single-valued branch, $\log f$, in $M \backslash \operatorname{supp} \sigma_{f}$ and the exterior differential of $\log f$, regarded as a 0 -current in $M$ with jumps taken into account, is

$$
d \log f=\frac{d f}{f}-2 \pi \mathrm{i} d H_{\sigma_{f}} .
$$

Since $\frac{d f}{f} \wedge \frac{d g}{g}=0$ the lemma combined with Theorem 2 gives the following alternative formula for the resultant.

COROLlary 1. With notations as above

$$
\mathcal{R}(f, g)=\exp \left(-\int_{M} \frac{d f}{f} \wedge d H_{\sigma_{g}}\right)=\exp \int_{\sigma_{g}} \frac{d f}{f} .
$$


In the corollary $\sigma_{f}$ may be replaced by any 1-chain $\gamma$ with $\partial \gamma=(g)$, because this will make a difference in the integral only by an integer multiple of $2 \pi \mathrm{i}$.

Next we compute

$$
\begin{aligned}
& d \log f \wedge d \log g=\left(\frac{d f}{f}-2 \pi \mathrm{i} d H_{\sigma_{f}}\right) \wedge\left(\frac{d g}{g}-2 \pi \mathrm{i} d H_{\sigma_{g}}\right) \\
& =\frac{d f}{f} \wedge d \log g+d \log f \wedge \frac{d g}{g}+(2 \pi \mathrm{i})^{2} d H_{\sigma_{f}} \wedge d H_{\sigma_{g}} .
\end{aligned}
$$

The integral of $d \log f \wedge d \log g=d(\log f \wedge d \log g)$ over $M$ is zero because $M$ is closed, and the integral of the last member, $(2 \pi \mathrm{i})^{2} d H_{\sigma_{f}} \wedge d H_{\sigma_{g}}$, is an integer multiple of $(2 \pi \mathrm{i})^{2}$. Therefore, after integration and taking the exponential we get

$$
\exp \left[\frac{1}{2 \pi \mathrm{i}} \int_{M} \frac{d f}{f} \wedge d \log g+\frac{1}{2 \pi \mathrm{i}} \int_{M} d \log f \wedge \frac{d g}{g}\right]=1 .
$$

This proves the symmetry:

COROllary 2. Let $f$ and $g$ be two meromorphic functions on a closed Riemann surface with disjoint divisors. Then

$$
\mathcal{R}(f, g)=\mathcal{R}(g, f) .
$$

REMARK 2. This symmetry is also a consequence of Weil's reciprocity law [55] (see Section 9 for further details), and may alternatively be proved, in a more classical fashion, by evaluating the integral in Cauchy's formula $\int_{\partial M^{\prime}} \log f \wedge d \log g=0$ (cf. [20, p. 242]). It is also obtained by directly evaluating the last integral in (32).

REMARK 3. If the divisors of $f$ and $g$ are not disjoint but $f, g$ still form an admissible pair, then both $\mathcal{R}(f, g)$ and $\mathcal{R}(g, f)$ are either 0 or $\infty$, hence the symmetry remains valid although in a degenerate way. In this case, and more generally for nonadmissible pairs, Weil's reciprocity law in the form (77) (in Section 9) contains more information.

By conjugating $g$ one gets the following formula for the modulus of the resultant in terms of a Dirichlet integral.

THEOREM 3. Let $f$ and $g$ be two meromorphic functions on a compact Riemann surface whose divisors have disjoint supports. Then

$$
|\mathcal{R}(f, g)|^{2}=\exp \left[\frac{1}{2 \pi \mathrm{i}} \int_{M} \frac{d f}{f} \wedge \frac{d \bar{g}}{\bar{g}}\right]
$$

Proof. By Lemma 2 we have

$\frac{1}{2 \pi \mathrm{i}} d \log f \wedge d \log \bar{g}=\frac{1}{2 \pi \mathrm{i}} \frac{d f}{f} \wedge \frac{d \bar{g}}{\bar{g}}+\frac{d f}{f} \wedge d H_{\sigma_{g}}-d H_{\sigma_{f}} \wedge \frac{d \bar{g}}{\bar{g}}-2 \pi \mathrm{i} d H_{\sigma_{f}} \wedge d H_{\sigma_{g}}$.

Integrating over $M$ and taking the exponential yields, in view of (32), the required formula. 


\section{Potential theoretic interpretations}

5.1. The mutual energy and the resultant. We recall some potential theoretic concepts (see, e.g., [41] for more details). The potential of a signed measure ("charge distribution") $\mu$ with compact support in $\mathbb{C}$ is

$$
U^{\mu}(z)=-\int \log |z-\zeta| d \mu(\zeta)
$$

The mutual energy between two such measures, $\mu$ and $\nu$, is (when defined)

$$
I(\mu, \nu)=-\iint \log |z-\zeta| d \mu(z) d \nu(\zeta)=\int U^{\mu} d \nu=\int U^{\nu} d \mu,
$$

and the energy of $\mu$ itself is $I(\mu)=I(\mu, \mu)$. In case $\int d \nu=\int d \mu=0$ the above mutual energy can after partial integration be written as a Dirichlet integral:

$$
I(\mu, \nu)=\frac{1}{2 \pi} \int d U^{\mu} \wedge * d U^{\nu}
$$

where $*$ is the Hodge star.

If $K \subset \mathbb{C}$ is a compact set then either $I(\mu)=+\infty$ for all $\mu \geq 0$ with $\operatorname{supp} \mu \subset K, \int d \mu=1$, or there is a unique such measure for which $I(\mu)$ has a finite minimum value. In the latter case $\mu$ is called the equilibrium distribution for $K$ because its potential is constant on $K$ (except possibly for a small exceptional set), say

$$
U^{\mu}=\gamma \text { (const) on } K \text {. }
$$

The logarithmic capacity of $K$ is defined as

$$
\operatorname{cap}(K)=e^{-\gamma}=e^{-I(\mu)} .
$$

(If $I(\mu)=+\infty$ for all $\mu$ as above then $\operatorname{cap}(K)=0$ ).

Now let us think of signed measures as (special cases of) 2 -form currents. Then, for example, (31) associates to each divisor $D$ in $\mathbb{C}$ the charge distribution $\mu=\mu_{D}$. In particular, for any rational function $f$ of the form $f(z)=\prod_{i=1}^{m} \frac{z-a_{i}}{z-b_{i}}$ we have the charge distribution

$$
\mu=\mu_{(f)}=\sum_{i=1}^{m} \delta_{a_{i}} d x \wedge d y-\sum_{i=1}^{m} \delta_{b_{i}} d x \wedge d y,
$$

the potential of which is $U^{\mu}=-\log |f|$.

One point we wish to make is that the resultant of two rational functions, $f$ and $g$, relates in the same way to the mutual energy. In fact, with $\mu=\mu_{(f)}$ and $\nu=\mu_{(g)}$,

$|\mathcal{R}(f, g)|^{2}=\exp [\langle(f), \log g\rangle+\langle(f), \overline{\log g}\rangle]=e^{2\langle(f), \log |g|\rangle}=e^{-2 \int U^{\nu} d \mu}=e^{-2 I(\mu, \nu)}$,

hence

$$
I(\mu, \nu)=-\log |\mathcal{R}(f, g)| .
$$

The Dirichlet integral (34) for $I(\mu, \nu)$ essentially gives the link between (35) and (33). 
5.2. Discriminant. Recall that the (polynomial) discriminant $\operatorname{Dis}_{\text {pol }}(f)$ is a polynomial in the coefficients of $f$ which vanishes whenever $f$ has a multiple root. In case of a monic polynomial $f(z)=\prod_{i=1}^{m}\left(z-a_{i}\right)$ we have

$$
\operatorname{Dis}_{\text {pol }}(f)=(-1)^{\frac{m(m-1)}{2}} \mathcal{R}_{\text {pol }}\left(f, f^{\prime}\right)=\prod_{i<j}\left(a_{i}-a_{j}\right)^{2} .
$$

Thus the discriminant is the square of the Van der Monde determinant.

The discriminant can be related to a renormalized self-energy of the measure $\mu=\mu_{(f)}$. The self-energy itself is actually infinite because point charges always have infinite energy. Formally:

$$
I(\mu)=\int U^{\mu} d \mu=\langle(f),-\log |f|\rangle=-\log \prod_{i, j=1}^{m}\left|a_{i}-a_{j}\right| \quad(=+\infty) .
$$

The renormalized energy $\widehat{I}(\mu)$ is obtained by simply subtracting off the infinities $I\left(\delta_{a_{i}}\right)$, i.e., the diagonal terms above:

$$
\widehat{I}(\mu)=-\log \prod_{i \neq j}\left|a_{i}-a_{j}\right|=-\log \prod_{i<j}\left|a_{i}-a_{j}\right|^{2}=-\log \left|\operatorname{Dis}_{\mathrm{pol}}(f)\right| .
$$

Thus, $\left|\operatorname{Dis}_{\operatorname{pol}}(f)\right|=e^{-\widehat{I}(\mu)}$. Here $\int d \mu=\operatorname{deg} f=m$, and after normalization (there are $m(m-1)$ factors in $\left.\operatorname{Dis}_{\text {pol }}(f)\right)$ it is known that the transfinite diameter

$$
d_{\infty}(K)=\lim _{m \rightarrow \infty} \max _{\operatorname{deg} f=m}\left|\operatorname{Dis}_{\mathrm{pol}}(f)\right|^{\frac{1}{m(m-1)}},
$$

equals the capacity: $d_{\infty}(K)=\operatorname{cap}(K)$.

Notice also that the discriminant may be regarded as a renormalized self-resultant $\mathcal{R}_{\text {pol }}(f, f)$ :

$$
\mathcal{R}_{\mathrm{pol}}(f, f)=\prod_{i, j}\left(a_{i}-a_{j}\right) \stackrel{\text { renorm }}{\Longrightarrow} \operatorname{Dis}_{\mathrm{pol}}(f)=\prod_{i \neq j}\left(a_{i}-a_{j}\right) .
$$

We can use the same renormalization method to arrive at a definition of discriminant in the rational case. Let $f$ be a rational function

$$
f(z)=\frac{f_{1}(z)}{f_{2}(z)} \equiv \frac{\prod_{i=1}^{m}\left(z-a_{i}\right)}{\prod_{i=1}^{m}\left(z-b_{i}\right)} .
$$

Then applying the scheme in (36) gives

$$
\begin{aligned}
\mathcal{R}(f, f)=\prod_{i, j} \frac{\left(a_{i}-a_{j}\right)\left(b_{i}-b_{j}\right)}{\left(a_{i}-b_{j}\right)\left(b_{i}-a_{j}\right)} \stackrel{\text { renorm }}{\Longrightarrow} \\
\stackrel{\prod_{i \neq j}\left(a_{i}-a_{j}\right) \prod_{i \neq j}\left(b_{i}-b_{j}\right)}{\Longrightarrow} \operatorname{Dis}(f):=\frac{\mathcal{R}_{\mathrm{pol}}\left(f_{1}, f_{1}^{\prime}\right) \mathcal{R}_{\mathrm{pol}}\left(f_{2}, f_{2}^{\prime}\right)}{\prod_{i, j}\left(a_{i}-b_{j}\right) \prod_{i, j}\left(b_{i}-a_{j}\right)}
\end{aligned}
$$

The corresponding renormalized energy of $\mu=\mu_{(f)}$ is

$$
\widehat{I}(\mu)=-\log \left|\frac{\prod_{i \neq j}\left(a_{i}-a_{j}\right) \prod_{i \neq j}\left(b_{i}-b_{j}\right)}{\prod_{i, j}\left(a_{i}-b_{j}\right) \prod_{i, j}\left(b_{i}-a_{j}\right)}\right|=-\log |\operatorname{Dis}(f)|
$$

which yields

$$
|\operatorname{Dis}(f)|=e^{-\widehat{I}(\mu)} .
$$


We note that the definition (37) of $\operatorname{Dis}(f)$ is consistent with the so-called characteristic property of the polynomial discriminant [19, p. 405]. Namely, one can easily verify that the meromorphic resultant of two rational functions can be obtained as the polarization of the discriminant in (37), that is

$$
\mathcal{R}(f, g)^{2}=\frac{\operatorname{Dis}(f g)}{\operatorname{Dis}(f) \operatorname{Dis}(g)} .
$$

5.3. Riemann surface case. Much of the above can be repeated for an arbitrary compact Riemann surface $M$. For any signed measure $\mu$ on $M$ with $\int_{M} d \mu=0$ there is potential $U^{\mu}$, uniquely defined up to an additive constant, such that

$$
-d * d U^{\mu}=2 \pi \mu .
$$

Here $\mu$ is considered as a 2-form current ( $\mu$ may actually be an arbitrary 2form current with $\langle\mu, 1\rangle=0$, and then $U^{\mu}$ will be a 0 -current; the existence and uniqueness of $U^{\mu}$ follows from ordinary Hodge theory, see e.g. [20, p. 92]).

The mutual energy between two measures as above can still be defined as

$$
I(\mu, \nu)=\int U^{\mu} d \nu=\int U^{\nu} d \mu
$$

and (34) remains true. Similarly, (35) remains valid for $\mu=\mu_{(f)}, \nu=\mu_{(g)}$. Thus

$$
|\mathcal{R}(f, g)|=e^{-I(\mu, \nu)} .
$$

It is interesting to notice that this gives a way of defining the modulus of the resultant of any two divisors of degree zero: if $\operatorname{deg} D_{1}=\operatorname{deg} D_{2}=0$ with supp $D_{1} \cap \operatorname{supp} D_{2}=\emptyset$ then one naturally sets

$$
\left|\mathcal{R}\left(D_{1}, D_{2}\right)\right|=e^{-I\left(\mu_{D_{1}}, \mu_{D_{2}}\right)} .
$$

It is not clear whether there is any natural definition of $\mathcal{R}\left(D_{1}, D_{2}\right)$ itself, except in genus zero where we have (17). Directly from the definition (23) we can however define $\mathcal{R}(D, g)=g(D)$ for $D$ a divisor of degree zero and $g$ a meromorphic function.

\section{The resultant as a function of the quotient}

6.1. Resultant identities. In previous sections we have considered the resultant as a function of two meromorphic functions, $f$ and $g$, say. Sometimes, however, it is possible and convenient to think of the resultant as a function of just one function, namely the quotient $h=\frac{f}{g}$. In general, part of the information about $f$ and $g$ is lost in $h$, hence some additional information has to be provided.

For instance, if $f$ and $g$ are two monic polynomials, then formula (14) in its simplest form, when $N=n$, reads

$$
\mathcal{R}_{\mathrm{pol}}(f, g)=\operatorname{det} t_{m, n}(h) .
$$

Another example is if the divisors of $f$ and $g$ are confined to lie in prescribed disjoint sets: given any set $U \subset M$ then among pairs $f, g$ with $\operatorname{supp}(f) \subset U, \operatorname{supp}(g) \subset M \backslash U$, the resultant $\mathcal{R}(f, g)$ only depends on $\frac{f}{g}$. 
Integral representations for $\mathcal{R}(f, g)$ in terms of only $f / g$ and $U$ will in such cases be elaborated in Section 6.2 (Theorem 4).

In the remaining part of this section we shall pursue a further point of view. Suppose that the divisors of $f$ and $g$ are not necessarily disjoint but that $f$ and $g$ still form an admissible pair. In general we have, with $h=f / g$,

$$
\text { ord } h \leq \text { ord } f+\text { ord } g,
$$

and it is easy to see that $\mathcal{R}(f, g)=0$ if and only if this inequality is strict (because strict inequality means that at least one common zero or one common pole of $f, g$ cancels out in the quotient $f / g$ ).

Now start with $h$ and consider admissible pairs $f, g$ with $h=f / g$ and such that

$$
\operatorname{ord} h=\operatorname{ord} f+\operatorname{ord} g \text {. }
$$

In general there are many such pairs $f, g$ and by the above $\mathcal{R}(f, g) \neq 0$ for all of them. The question we want to consider is whether there are any restrictions on which values $\mathcal{R}(f, g)$ can take. At least in the rational case there turns out be such restrictions and this is what we call resultant identities.

Let $d \geq 1$ and

$$
h(z)=\prod_{i=1}^{d} \frac{z-a_{i}}{z-b_{i}} .
$$

Let $C_{d}^{m}$ denote the set of all increasing length- $m$ sequences $\left(i_{1}, \ldots, i_{m}\right)$, $1 \leq i_{1}<\ldots<i_{m} \leq d$. For two given elements $I, J \in C_{d}^{m}$ define

$$
h_{I J}(z)=\frac{\prod_{i \in I}\left(z-a_{i}\right)}{\prod_{j \in J}\left(z-b_{j}\right)},
$$

Then all the solutions $f, g$ of (38), up to a constant factor (which by (24) is inessential for the resultant), are parameterized by

$$
f(z)=h_{I J}(z), \quad g(z)=\frac{h_{I J}(z)}{h(z)}=\frac{1}{h_{I^{\prime} J^{\prime}}(z)},
$$

where the prime denotes complement, e.g., $I^{\prime}=\{1, \ldots, d\} \backslash I$.

The main observation of this section is that the resultants $\mathcal{R}(f, g)$ satisfy a system of linear identities. An extended version of the material below with applications to rational and trigonometric identities will appear in [27].

Proposition 2. Let $0 \leq m \leq d$ and $J \in C_{d}^{m}$. Then

$$
\sum_{I \in C_{d}^{m}} \mathcal{R}\left(h_{I J}, 1 / h_{I^{\prime} J^{\prime}}\right)=\sum_{I \in C_{d}^{m}} \mathcal{R}\left(h_{J I}, 1 / h_{J^{\prime} I^{\prime}}\right)=1 .
$$

Proof. We briefly describe the idea of the proof. Denote by $\mathbf{A}$ and $\mathbf{B}$ the two Van der Monde matrices with entries $\left(a_{i}^{j-1}\right)$ and $\left(b_{i}^{j-1}\right), 1 \leq i, j \leq d$, respectively. Let $I=\left\{i_{1}, \ldots, i_{m}\right\}$ and $J=\left\{j_{1}, \ldots, j_{m}\right\}$. Then one can readily show that

$$
\mathcal{R}\left(h_{I J}, 1 / h_{I^{\prime} J^{\prime}}\right)=(-1)^{n} \operatorname{det} \Lambda_{I J} \operatorname{det}\left(\Lambda^{-1}\right)_{I J},
$$

where $n=\sum_{s=1}^{m}\left(i_{s}+j_{s}\right)$. Here $\Lambda=\mathbf{A B}^{-1}$ and $\Lambda_{I J}\left(\operatorname{resp} .\left(\Lambda^{-1}\right)_{I J}\right)$ denotes the minor of $\Lambda$ (resp. $\Lambda^{-1}$ ) formed by intersection of the rows $i \in I$ and 
the columns $j \in J$. Hence the required identities follow from (41) and the Laplace expansion theorem for determinants.

In the simplest case, $d=2, m=1,(40)$ amounts to the characteristic property of the cross-ratio:

$$
(a, b, c, d)+(a, c, b, d)=1 .
$$

The resultants in (40) appear also in the so-called Day's formula [12] for the determinants of truncated Toeplitz operators. Let $h$ be a function given by (39) such that $\left|b_{i}\right| \neq 1$ for all $i$, and let $J=\left\{j:\left|b_{j}\right|>1\right\}$.

Introduce the Toeplitz matrix of order $N$

$$
t_{N}(h) \equiv\left(\begin{array}{cccc}
h_{0} & h_{-1} & \ldots & h_{1-N} \\
h_{1} & h_{0} & \ldots & h_{2-N} \\
\ldots & \ldots & \ldots & \ldots \\
h_{N-1} & h_{N-2} & \ldots & h_{0}
\end{array}\right)
$$

where $h_{k}=\frac{1}{2 \pi} \int_{0}^{2 \pi} e^{-\mathrm{i} k \theta} h\left(e^{\mathrm{i} \theta}\right) d \theta$ are the Fourier coefficients of $h$ on the unit circle. Then, in our notation, Day's formula reads

$$
\operatorname{det} t_{N}(h)=\sum_{I \in C_{d}^{m}} \mathcal{R}\left(h_{I J}, 1 / h_{I^{\prime} J^{\prime}}\right) \cdot h_{I^{\prime} J^{\prime}}^{N}(0),
$$

where $m$ denotes the cardinality of $J$ and $N \geq 1$. Notice that formal substitution of $N=0$ with $t_{0}(h)=1$ into (43) gives exactly the statement of Proposition 2.

REMARK 4. Taking double sums in (40) (over all $I, J \in C_{d}^{m}$ ) we get quantities which occur also when computing subresultants (see, e.g., [32]). Recall that the (scalar) subresultant of degree $k$ is the determinant of the matrix obtained from the Sylvester matrix (12) by deleting the last $2 k$ rows and the last $k$ columns with coefficients of $f$, and the last $k$ columns with coefficients of $g$. In a different context, the subresultants are determinants of certain submatrices of the Sylvester matrix (12) which occur as successive remainders in finding the greatest common divisor of two polynomials by the Euclid algorithm [50].

The identities (40) have beautiful trigonometric interpretations. Take

$$
f(z)=\prod_{k=1}^{m} \frac{z-e^{2 \mathrm{i} a_{k}}}{z-e^{2 \mathrm{i} b_{k}}}, \quad g(z)=\prod_{l=1}^{n} \frac{z-e^{2 \mathrm{i} c_{l}}}{z-e^{2 \mathrm{i} d_{l}}} .
$$

Then one easily finds that

$$
\mathcal{R}(f, g)=\prod_{k=1}^{m} \prod_{l=1}^{n} \frac{\sin \left(a_{k}-c_{l}\right)}{\sin \left(a_{k}-d_{l}\right)} \frac{\sin \left(b_{k}-d_{l}\right)}{\sin \left(b_{k}-c_{l}\right)},
$$

hence a direct application of (40) gives the following.

Corollary 3. Let $d \geq 2$ and $J \in C_{d}^{m}$. Then

$$
\sum_{I} \frac{\prod_{i, j^{\prime}} \sin \left(a_{i}-b_{j^{\prime}}\right) \prod_{i^{\prime}, j^{\prime}} \sin \left(b_{j}-a_{i^{\prime}}\right)}{\prod_{i, i^{\prime}} \sin \left(a_{i}-a_{i^{\prime}}\right) \prod_{j, j^{\prime}} \sin \left(b_{j}-b_{j}^{\prime}\right)}=1,
$$

where the sum is taken over all subsets $I \in C_{d}^{m}$ and the product over $i \in I$, $i^{\prime} \in I^{\prime}, j \in J, j^{\prime} \in J^{\prime}$. 
For example, specializing by taking $b_{j}=\frac{\pi}{2}+a_{i}$ in (44) one gets identities in the spirit of those given recently in $[7],[8]$.

There are also analogues of Proposition 2 for the complex torus $M=$ $\mathbb{C} / L_{\tau}$. For these one has to take into account the Abel condition (30). Although we have not been able to find complete analogues of the rational resultant identities, one particular case is worth mentioning here. Notice that the minimal possible value of $d$ in order for a meromorphic function $h(z)=\prod_{i=1}^{d} \frac{\theta\left(z-u_{i}\right)}{\theta\left(z-v_{i}\right)}$ to split into two non-constant meromorphic functions, i.e. $h=f / g$, is $d=4$. One can readily show that any such function may be written as

$$
h(z)=\frac{\phi\left(z-z_{0}, a_{1}\right) \phi\left(z-z_{0}, a_{2}\right)}{\phi\left(z-z_{0}, b_{1}\right) \phi\left(z-z_{0}, b_{2}\right)}
$$

where $\phi(\zeta, a)=\theta(\zeta-a) \theta(\zeta+a)$. We additionally assume that $a_{1} \pm a_{2} \notin L$ and $b_{1} \pm b_{2} \notin L$. Then all non-constant solutions of (38) are given by

$$
f(z)=\frac{\phi\left(z, a_{i}\right)}{\phi\left(z, b_{j}\right)}, \quad g(z)=\frac{\phi\left(z, b_{j^{\prime}}\right)}{\phi\left(z, a_{i^{\prime}}\right)}, \quad i, j=1,2,
$$

where $\left\{k, k^{\prime}\right\}=\{1,2\}$. Hence

$$
\rho_{i j}:=\mathcal{R}(f, g)=\left[\frac{\theta\left(a_{i}-b_{j^{\prime}}\right) \theta\left(a_{i}+b_{j^{\prime}}\right) \theta\left(a_{i^{\prime}}-b_{j}\right) \theta\left(a_{i^{\prime}}+b_{j}\right)}{\theta\left(a_{i}-a_{i^{\prime}}\right) \theta\left(a_{i}+a_{i^{\prime}}\right) \theta\left(b_{j}-b_{j^{\prime}}\right) \theta\left(b_{j}+b_{j^{\prime}}\right)}\right]^{2},
$$

and there only two different values of $\rho_{i j}$ :

$$
\xi_{1}:=\rho_{11}=\rho_{22}, \quad \xi_{2}:=\rho_{12}=\rho_{21} .
$$

Using the famous addition theorem of Weierstraß

$$
\begin{aligned}
0 & =\theta(a-c) \theta(a+c) \theta(b-d) \theta(b+d)-\theta(a-b) \theta(a+b) \theta(c-d) \theta(c+d) \\
& -\theta(a-d) \theta(a+d) \theta(b-c) \theta(b+c),
\end{aligned}
$$

one finds that (with appropriate choices of signs)

$$
\pm \sqrt{\xi_{1}} \pm \sqrt{\xi_{2}}=1
$$

or more adequately: $\left(1-\xi_{1}\right)^{2}+\left(1-\xi_{2}\right)^{2}=2 \xi_{1} \xi_{2}$.

The identity (45) may be generalized to functions of the kind

$$
h(z)=\prod_{k=1}^{d} \frac{\phi\left(z-z_{0}, a_{k}\right)}{\phi\left(z-z_{0}, b_{k}\right)} .
$$

However the problem of description of the range of $\mathcal{R}(f, g)$ in (38) for general meromorphic functions $h$ on $\mathbb{C} / L_{\tau}$ remains open.

6.2. Integral representation of $\mathcal{R}_{U}$. Let us now turn to the situation of having a preassigned set $U \subset M$ and consider resultants $\mathcal{R}(f, g)$ for meromorphic functions $f$ and $g$ with $\operatorname{supp}(f) \subset U, \operatorname{supp}(g) \subset M \backslash U$. It is easy to see that for such pairs $\mathcal{R}(f, g)$ only depends on the quotient $h=$ $\mathrm{f} / \mathrm{g}$. Indeed, this is obvious from the fact (see (24)) that the resultant only depends on the divisors: under the above assumptions the divisors of $f$ and $g$ are clearly determined by $h$ and $U$.

To make the above in a slightly more formal we may define $\mathcal{R}\left(D_{1}, D_{2}\right)$ for any two principal divisors $D_{1}, D_{2}$ having, e.g., disjoint supports. For any divisor $D$, let $D_{U}$ denote its restriction to the set $U$ and extended by 
zero outside $U$ (thus with $\left.D=\sum_{a \in M} D(a) a, D_{U}=\sum_{a \in U} D(a) a\right)$. Then in the situation at hand we can write

$$
\mathcal{R}(f, g)=\mathcal{R}((f),(g))=\mathcal{R}\left((h)_{U},(h)_{U}-(h)\right),
$$

which only depends on $h$ and $U$. This motivates the following definition.

Definition 3. For any set $U \subset M$ and any meromorphic function $h$ on $M$ such that $(h)_{U}$ is a principal divisor we define

$$
\mathcal{R}_{U}(h)=\mathcal{R}\left((h)_{U},(h)_{U}-(h)\right) .
$$

It is easy to check that

$$
\mathcal{R}_{U}(h)=\mathcal{R}_{M \backslash U}(h) .
$$

We shall consider the symmetric situation that

$$
M=U \cup \Gamma \cup V,
$$

where $U, V$ are disjoint nonempty open sets and $\Gamma=\partial U=\partial V$. We provide $\Gamma$ with the orientation of $\partial U$. By the above, with $f$ and $g$ meromorphic on $M, \operatorname{supp}(f) \subset U, \operatorname{supp}(g) \subset V$ and $h=f / g$ we have

$$
\mathcal{R}_{U}(h)=\mathcal{R}_{V}(h)=\mathcal{R}(f, g) .
$$

Note that the function $h$ is holomorphic and nonzero in a neighbourhood of $\Gamma, h \in \mathcal{O}^{*}(\Gamma)$, and that it is uniquely defined by its values on $\Gamma$. Our aim is to find an integral representation for $\mathcal{R}_{U}(h)$ in terms only of the values of $h$ on $\Gamma$.

The problem of decomposing a given $h \in \mathcal{O}^{*}(\Gamma)$ into functions $f \in$ $\mathcal{O}^{*}(\bar{V}), g \in \mathcal{O}^{*}(\bar{U})$ with $h=f / g$ is a special case of the second Cousin problem. By taking logarithms we shall reduce it, under symplifying assumptions, to the corresponding additive problem, which is the first Cousin problem. For the latter we have the following simple criterion for solvability.

Lemma 3. Let $M=U \cup \Gamma \cup V$ be as above. Necessary and sufficient condition for a function $H \in \mathcal{O}(\Gamma)$ to be decomposable as

$$
H=H_{+}-H_{-} \quad \text { on } \Gamma
$$

with $H_{+} \in \mathcal{O}(\bar{U}), H_{-} \in \mathcal{O}(\bar{V})$ is that

$$
\int_{\Gamma} H \wedge \omega=0 \quad \text { for all } \omega \in \mathcal{O}^{1,0}(M) .
$$

When the decomposition exists the functions $H_{ \pm}$are unique up to addition of a common constant (more adequately: a function in $\mathcal{O}(M)$ ).

The lemma is well-known and can be deduced for example from the Serre duality theorem. We shall just remark that "explicit" representations of $H_{ \pm}$ can be given in terms of a suitable Cauchy kernel:

$$
H_{ \pm}(z)=\frac{1}{2 \pi \mathrm{i}} \int_{\Gamma} H(\zeta) \Phi\left(z, \zeta ; z_{0}, \zeta_{0}\right) d \zeta
$$

the plus sign for $z \in U$, minus for $z \in V$. The kernel $\Phi\left(z, \zeta ; z_{0}, \zeta_{0}\right)$ is, in the variable $z$, a meromorphic function with a simple pole at $z=\zeta$ and a pole of higher order (depending on the genus) at $z=\zeta_{0}$. In the variable $\zeta$ it is a meromorphic one-form with simple poles of residues plus and minus one 
at $\zeta=z$ and $\zeta=z_{0}$ respectively; $z_{0}$ and $\zeta_{0}$ are fixed but arbitrary points, $z_{0} \neq \zeta_{0}$. In the case of the Riemann sphere, $\Phi\left(z, \zeta ; z_{0}, \zeta_{0}\right) d \zeta$ is the ordinary Cauchy kernel

$$
\Phi\left(z, \zeta ; z_{0}, \zeta_{0}\right) d \zeta=\frac{d \zeta}{\zeta-z}-\frac{d \zeta}{\zeta-z_{0}}
$$

hence does not involve $\zeta_{0}$. In the the case of higher genus the point $\zeta_{0}$ is really needed. We refer to $[\mathbf{4 0}]$ for the construction of the Cauchy kernel in general.

THEOREM 4. Let $M=U \cup \Gamma \cup V$ with $U$ connected and simply connected, and let $h$ be meromorphic on $M$ without poles and zeros on $\Gamma$. Assume in addition that

$$
\frac{1}{2 \pi \mathrm{i}} \int_{\Gamma} \frac{d h}{h}=0
$$

and that

$$
\int_{\Gamma} \log h \wedge \omega=0 \quad \text { for all } \omega \in \mathcal{O}^{1,0}(M)
$$

(the previous condition guarantees that a single-valued branch of $\log h$ exists on $\Gamma)$. Then $(h)_{U}$ is a principal divisor and

$$
\mathcal{R}_{U}(h)=\exp \left[\frac{1}{2 \pi \mathrm{i}} \int_{\Gamma} d(\log h)_{-} \wedge(\log h)_{+}\right] .
$$

REMARK 5. Ideally (48) should be replaced be the weaker condition that there exists a closed 1-chain $\gamma$ on $M$ such that

$$
\int_{\Gamma} \log h \wedge \omega=2 \pi \mathrm{i} \int_{\gamma} \omega \quad \text { for all } \omega \in \mathcal{O}^{1,0}(M) .
$$

In fact, this turns out to be exactly, by Abel's theorem, the necessary and sufficient condition for $(h)_{U}$ to be a principal divisor. However, (49) would lead to a more complicated formula for $\mathcal{R}_{U}(h)$. Note that (48) is vacuously satisfied in the case $M=\mathbb{P}$, which will be our main application. Condition (47) says that the divisor $(h)_{U}$ has degree zero.

Proof. We first prove that $(h)_{U}$ is a principal divisor. Using the notation of Lemma 2 we make $\log h$ into a single-valued function on all of $M$ by making cuts along a 1-chain $\sigma_{h}$ such that $\partial \sigma_{h}=(h)$. Since $\log h$ is already single-valued on $\Gamma, \sigma_{h}$ can be chosen not to intersect $\Gamma$. Thus $\sigma_{h}$ consists of two disjoint parts, $\sigma_{h} \cap U$ and $\sigma_{h} \cap V$. The terms of $\sigma_{h}$ containing the curves $\alpha_{1}, \ldots, \beta_{p}$ will appear in $\sigma_{h} \cap V$ because $U$ is simply connected.

Now, for all $\omega \in \mathcal{O}^{1,0}(M)$ we have by (48) and Lemma 2

$$
\begin{aligned}
0 & =\frac{1}{2 \pi \mathrm{i}} \int_{\Gamma} \log h \wedge \omega=\frac{1}{2 \pi \mathrm{i}} \int_{U} d \log h \wedge \omega=\frac{1}{2 \pi \mathrm{i}} \int_{U}\left(\frac{d h}{h}-2 \pi \mathrm{i} d H_{\sigma_{h}}\right) \wedge \omega \\
& =\frac{1}{2 \pi \mathrm{i}} \int_{U} \frac{d h}{h} \wedge \omega-\int_{U} d H_{\sigma_{h}} \wedge \omega=-\int_{M} d H_{\sigma_{h} \cap U} \wedge \omega=-\int_{\sigma_{h} \cap U} \omega .
\end{aligned}
$$

By Abel's theorem this implies that $\partial\left(\sigma_{h} \cap U\right)=(h)_{U}$ is a principal divisor (condition (49), in place of (48), would have been enough for this conclusion).

The divisor $(h)_{U}$ being principal means that $(h)_{U}=(f)$ for some $f$ meromorphic on $M$. Setting $g=f / h$ we have $\operatorname{supp}(f) \subset U, \operatorname{supp}(g) \subset V$ 
and $h=f / g$. It follows that $\mathcal{R}_{U}(h)=\mathcal{R}(f, g)$, hence to prove the theorem it is by Theorem 2 enough to prove that

$$
\int_{\Gamma} d(\log h)_{-} \wedge(\log h)_{+}=\int_{M} \frac{d f}{f} \wedge d \log g .
$$

To that end we shall compare two decompositions of $d \log h=\frac{d h}{h}$ on $\Gamma$ : from Lemma 3 we get

$$
d \log h=d(\log h)_{+}-d(\log h)_{-} \quad \text { on } \Gamma
$$

with $(\log h)_{+} \in \mathcal{O}(\bar{U}),(\log h)_{-} \in \mathcal{O}(\bar{V})$, while $h=f / g$ gives

$$
\frac{d h}{h}=\frac{d f}{f}-\frac{d g}{g} \quad \text { on } \Gamma,
$$

where $d f / f \in \mathcal{O}^{1,0}(\bar{V}), d g / g \in \mathcal{O}^{1,0}(\bar{U})$.

It follows that

$$
\frac{d f}{f}+d(\log h)_{-}=\frac{d g}{g}+d(\log h)_{+} \quad \text { on } \Gamma
$$

and that the left and right members combine into a global 1-form $\omega_{0} \in$ $\mathcal{O}^{1,0}(M)$. Thus

$$
d(\log h)_{-}=\omega_{0}-\frac{d f}{f} \text { in } V, \quad d(\log h)_{+}=\omega_{0}-\frac{d g}{g} \text { in } U .
$$

In the simply connected domain $U$ we may write $\omega_{0}=d \varphi$ for some $\varphi \in \mathcal{O}(\bar{U})$ and also $\frac{d g}{g}=d \log g\left(d H_{\sigma_{g}}=0\right.$ in $U$ because $\sigma_{g}$ can be chosen to be $\sigma_{h} \cap V$; similarly $\sigma_{f}$ can be chosen to be $\left.\sigma_{h} \cap U\right)$. It follows after integration and adjusting $\varphi$ by a constant that

$$
(\log h)_{+}=\varphi-\log g \quad \text { in } U .
$$

Now we finally obtain

$$
\begin{aligned}
& \int_{\Gamma} d(\log h)_{-} \wedge(\log h)_{+}=\int_{\Gamma}\left(\omega_{0}-\frac{d f}{f}\right) \wedge(\varphi-\log g)=-\int_{\Gamma} \frac{d f}{f} \wedge(\varphi-\log g) \\
& =\int_{V} \frac{d f}{f} \wedge d \log g-\int_{\Gamma}(d \log h+d \log g) \wedge \varphi=\int_{M} \frac{d f}{f} \wedge d \log g,
\end{aligned}
$$

as desired.

REMARK 6. Under the assumptions of the theorem, the solution of the second Cousin problem of finding $f, g$ such that $h=f / g$ on $\Gamma$ is given by

$$
\begin{array}{ll}
f=\exp \left[\int \frac{d f}{f}\right]=\exp \left[\int\left(\omega-d(\log h)_{-}\right)\right] & \text {in } V \\
g=\exp \left[\int \frac{d g}{g}\right]=\exp \left[\int\left(\omega-d(\log h)_{+}\right)\right] & \text {in } U
\end{array}
$$

(indefinite integrals), where $\omega \in \mathcal{O}^{1,0}(M)$ is to be chosen such that $\int(\omega-$ $\left.d(\log h)_{-}\right)$is single-valued in $V$ modulo multiples of $2 \pi$ i. 
6.3. Cohomological interpretations of the quotient. Let us give some interpretations of the above material in terms of Čech cohomology. Given $h \in \mathcal{O}^{*}(\Gamma)$, let $U_{1}, V_{1}$ be open neighbourhoods of $\bar{U}$ and $\bar{V}$, respectively, such that $h \in \mathcal{O}^{*}\left(U_{1} \cap V_{1}\right)$. Then $\left\{U_{1}, V_{1}\right\}$ is an open covering of $M$, and relative to this $h$ represents an element $[h]$ in $H^{1}\left(M, \mathcal{O}^{*}\right)$. It is wellknown $[\mathbf{2 1}],[\mathbf{1 6}]$ that $[h]=0$ as an element in $H^{1}\left(M, \mathcal{O}^{*}\right)$ if and only if $h$ is a coboundary already with respect to $\left\{U_{1}, V_{1}\right\}$, i.e., if and only if there exist $f \in \mathcal{O}^{*}\left(V_{1}\right)$ and $g \in \mathcal{O}^{*}\left(U_{1}\right)$ such that $h=f / g$ in $U_{1} \cap V_{1}$. If $h$ is meromorphic in $M$, then so are $f$ and $g$.

Similarly, a function $H \in \mathcal{O}(\Gamma)$ represents an element $[H]$ in $H^{1}(M, \mathcal{O})$, and $[H]=0$ if and only if there exist $F \in \mathcal{O}\left(U_{1}\right), G \in \mathcal{O}\left(V_{1}\right)$ (for some $\left.U_{1} \supset \bar{U}, V_{1} \supset \bar{V}\right)$ such that $H=F-G$ on $\Gamma$.

The spaces $H^{1}(M, \mathcal{O})$ and $H^{1}\left(M, \mathcal{O}^{*}\right)$ are related via the long exact sequence of cohomology groups which comes from the exponential map on the sheaf level: with $e(f)=\exp [2 \pi \mathrm{i} f]$ we have

$$
0 \rightarrow \mathbb{Z} \rightarrow \mathcal{O} \stackrel{e}{\rightarrow} \mathcal{O}^{*} \rightarrow 1
$$

hence

$$
\begin{aligned}
0 & \rightarrow H^{0}(M, \mathbb{Z}) \rightarrow H^{0}(M, \mathcal{O}) \rightarrow H^{0}\left(M, \mathcal{O}^{*}\right) \rightarrow H^{1}(M, \mathbb{Z}) \rightarrow \\
& \rightarrow H^{1}(M, \mathcal{O}) \stackrel{e}{\rightarrow} H^{1}\left(M, \mathcal{O}^{*}\right) \rightarrow H^{2}(M, \mathbb{Z}) \rightarrow 0 .
\end{aligned}
$$

From this we extract the exact sequence

$$
0 \rightarrow H^{1}(M, \mathcal{O}) / H^{1}(M, \mathbb{Z}) \stackrel{e}{\rightarrow} H^{1}\left(M, \mathcal{O}^{*}\right) \stackrel{c}{\rightarrow} H^{2}(M, \mathbb{Z}) \rightarrow 0 .
$$

Here $c$ is the map which associates to $[h] \in H^{1}\left(M, \mathcal{O}^{*}\right)$ its characteristic class, or Chern class, and it is readily verified that it is given by

$$
c([h])=\operatorname{wind}_{\Gamma} h=\frac{1}{2 \pi \mathrm{i}} \int_{\Gamma} \frac{d h}{h}=\operatorname{deg}(h)_{U} .
$$

If $c([h])=0$ then $[h]$ is in the range of $e$. If $\Gamma$ is connected then $\log h$ is single-valued on $\Gamma$ and the preimage of $[h]$ can be represented by $H=$ $\frac{1}{2 \pi \mathrm{i}} \log h$. However, if $\Gamma$ is not connected then the preimage of $[h]$ cannot always be represented by a function $H \in \mathcal{O}(\Gamma)$, one needs a finer covering of $M$ than $\left\{U_{1}, V_{1}\right\}$ to represent it. This is a drawback of the method using the decomposition $M=U \cup \Gamma \cup V$ in combination with the exp-log map and explains some of our extra assumptions in Theorem 4.

Assume nevertheless that the preimage of $[h] \in H^{1}\left(M, \mathcal{O}^{*}\right)($ with $c([h])=$ $0)$ can be represented by $H=\frac{1}{2 \pi \mathrm{i}} \log h \in \mathcal{O}(\Gamma)$. Then of course $[h]=0$ if $[H]=0$ as an element in $H^{1}(M, \mathcal{O})$, i.e., if $\int_{\Gamma} H \wedge \omega=0$ for all $\omega \in$ $\mathcal{O}^{1,0}(M)$. However, what exactly is needed for $[h]=0$ is by (50) only that $[H] \in H^{1}(M, \mathbb{Z})$, and this what is expressed in (49).

Since, for $H \in \mathcal{O}(\Gamma),[H]=0$ as an element in $H^{1}(M, \mathcal{O})$ if and only if $\int_{\Gamma} H \wedge \omega=0$ for all $\omega \in \mathcal{O}^{1,0}(M)$, the pairing

$$
(\omega, H) \mapsto \int_{\Gamma} H \wedge \omega
$$

descends to a bilinear map

$$
H^{0}\left(M, \mathcal{O}^{1,0}\right) \times H^{1}(M, \mathcal{O}) \rightarrow \mathbb{C}
$$


This map is in fact the Serre duality pairing $([\mathbf{4 4}],[\mathbf{2 1}])$ with respect to the covering $\left\{U_{1}, V_{1}\right\}$. Versions of the Serre duality with respect to more general coverings will be discussed in the next section.

6.4. Resultant via Serre duality. We now return to the general integral formula in Theorem 2, and interpret the exponent $\frac{1}{2 \pi \mathrm{i}} \int_{M} \frac{d f}{f} \wedge d \log g$ directly in terms of the Serre duality pairing, which in general also involves a line bundle or a divisor. With a divisor $D$, the pairing looks

$$
\langle,\rangle_{\text {Serre }}: H^{0}\left(M, \mathcal{O}_{D}^{1,0}\right) \times H^{1}\left(M, \mathcal{O}_{-D}\right) \rightarrow \mathbb{C},
$$

between meromorphic $(1,0)$-forms with divisor $\geq-D$ and (equivalence classes of) cocycles of meromorphic functions with divisor $\geq D$.

In our case, given two meromorphic functions $f$ and $g$, we choose $D \geq$ 0 to be the divisor of poles of $\frac{d f}{f}$ (or any larger divisor), so that $\frac{d f}{f} \in$ $\Gamma\left(M, \mathcal{O}_{D}^{1,0}\right)$. As for the other factor, $\log g$ defines an element, which we denote by $[\delta \log g]$, of $H^{1}\left(M, \mathcal{O}_{-D}\right)$ as follows. First, with $\gamma_{g}$ as in the beginning of Section 4.1, choose an open cover $\left\{U_{i}\right\}$ of $M$ consisting of simply connected domains $U_{i}$ satisfying

$$
\left(\operatorname{supp} D \cup \operatorname{supp} \gamma_{g}\right) \cap U_{i} \cap U_{j}=\emptyset \quad \text { whenever } i \neq j
$$

(in particular supp $\gamma_{g} \cap \partial U_{i}=\emptyset$ for all $i$ ). Second, choose for each $i$ a branch, $(\log g)_{i}$, of $\log g$ in $U_{i} \backslash \gamma_{g}$. Finally, define a cocycle $\left\{(\delta \log g)_{i j}\right\}$, to represent $[\delta \log g] \in H^{1}\left(M, \mathcal{O}_{-D}\right)$, by

$$
(\delta \log g)_{i j}=(\log g)_{i}-(\log g)_{j} \quad \text { in } U_{i} \cap U_{j} .
$$

There exist smooth sections $\psi_{i}$ over $U_{i}$, vanishing on $D$, such that

$$
(\delta \log g)_{i j}=\psi_{i}-\psi_{j} \quad \text { in } U_{i} \cap U_{j} .
$$

One may for example choose a smooth function $\rho: M \rightarrow[0,1]$ which vanishes in a neighbourhood of supp $D \cup \operatorname{supp} \gamma_{g}$ and equals one on each $U_{i} \cap U_{j}, i \neq j$ and define $\psi_{i}=\rho(\log g)_{i}$ in $U_{i}$. In any case, (51) shows that the $\psi_{i}$ satisfy

$$
\bar{\partial} \psi_{i}=\bar{\partial} \psi_{j} \quad \text { in } U_{i} \cap U_{j}
$$

so that $\left\{\bar{\partial} \psi_{i}\right\}$ defines a global $(0,1)$-form $\bar{\partial} \psi$ on $M$. The Serre pairing is then defined by

$$
\left\langle\frac{d f}{f},[\delta \log g]\right\rangle_{\text {Serre }}=\frac{1}{2 \pi \mathrm{i}} \int_{M} \frac{d f}{f} \wedge \bar{\partial} \psi .
$$

It is straightforward to check that the result $(\bmod 2 \pi \mathrm{i})$ does not depend upon the choices made, and that it $(\bmod 2 \pi \mathrm{i})$ agrees with $\int_{M} \frac{d f}{f} \wedge d \log g$.

A variant of the above is to consider the product $\frac{d f}{f} \wedge[\delta \log g]$ directly as an element in $H^{1}\left(M, \mathcal{O}^{1,0}\right)$, because there is a natural multiplication map

$$
H^{0}\left(M, \mathcal{O}_{D}^{1,0}\right) \times H^{1}\left(M, \mathcal{O}_{-D}\right) \rightarrow H^{1}\left(M, \mathcal{O}^{1,0}\right),
$$

and use the residue map (sum of residues; see [21], [16])

$$
\text { res : } H^{1}\left(M, \mathcal{O}^{1,0}\right) \rightarrow \mathbb{C} \text {. }
$$

Then one verifies that

$$
\operatorname{res}\left(\frac{d f}{f} \wedge[\delta \log g]\right)=\frac{1}{2 \pi \mathrm{i}} \int_{M} \frac{d f}{f} \wedge d \log g \quad(\bmod 2 \pi \mathrm{i}) .
$$


In summary we have

TheOREM 5. For any two meromorphic functions $f$ and $g$

$$
\mathcal{R}(f, g)=\exp \left(\left\langle\frac{d f}{f},[\delta \log g]\right\rangle_{\text {Serre }}\right)=\exp \left(\operatorname{res}\left(\frac{d f}{f} \wedge[\delta \log g]\right)\right) .
$$

The above expressions can be viewed as polarized and global versions of the torsor, or local symbol, as studied by P. Deligne, see in particular Example 2.8 in $[\mathbf{1 3}]$.

\section{Determinantal formulas}

7.1. Resultant via Szegö's strong limit theorem. In this section we show that the resultant of two rational functions on $\mathbb{P}$ admits several equivalent representations, among others as a Cauchy determinant and as a determinant of a truncated Toeplitz operator. We start with establishing a connection between resultants and Szegö's strong limit theorem.

Let us apply the results of the previous section to the case when

$$
M=\mathbb{P}, \quad U=\mathbb{D}, \quad V=\mathbb{P} \backslash \overline{\mathbb{D}}, \quad \Gamma=\mathbb{T} \equiv \partial \mathbb{D},
$$

and $h$ is holomorphic and nonvanishing in a neighbourhood of $\mathbb{T}$ with wind $\mathbb{T} h=$ 0 (equivalent to that $\log h$ has a single-valued branch on $\mathbb{T}$ in this case). Choose an arbitrary branch, $\log h$, and expand it in a Laurent series

$$
\log h(z)=\sum_{-\infty}^{\infty} s_{k} z^{k}
$$

Note that $s_{0}$ is determined modulo $2 \pi \mathrm{i} \mathbb{Z}$ only and that the $s_{k}$ also are the Fourier coefficients of $\log h\left(e^{\mathrm{i} \theta}\right)$ :

$$
s_{k}=(\log h)_{k}=\frac{1}{2 \pi} \int_{0}^{2 \pi} e^{-\mathrm{i} k \theta} \log h\left(e^{\mathrm{i} \theta}\right) d \theta .
$$

Then using the Cauchy kernel (46) with $z_{0}=\infty$ one gets

$$
(\log h)_{+}(z)=\sum_{k=0}^{\infty} s_{k} z^{k}, \quad(\log h)_{-}(z)=-\sum_{k=1}^{\infty} s_{-k} z^{-k}
$$

and $d(\log h)_{-}(z)=\sum_{k=1}^{\infty} k s_{-k} \frac{d z}{z^{k+1}}$. This gives the formula

$$
\mathcal{R}_{\mathbb{D}}(h)=\exp \left[\sum_{k=1}^{\infty} k s_{k} s_{-k}\right]
$$

In particular, we have the following corollary of Theorem 4 .

Corollary 4. Let $f$ and $g$ be two rational functions with $\operatorname{supp}(f) \subset \mathbb{D}$ and $\operatorname{supp}(g) \subset \mathbb{P} \backslash \overline{\mathbb{D}}$. Then

$$
\mathcal{R}(f, g)=\mathcal{R}_{\mathbb{D}}\left(\frac{f}{g}\right)=\exp \left[\sum_{k=1}^{\infty} k s_{k} s_{-k}\right]
$$

where $\log \frac{f\left(e^{\mathrm{i} \theta}\right)}{g\left(e^{\mathrm{i} \theta}\right)}=\sum_{k=-\infty}^{\infty} s_{k} e^{\mathrm{i} k \theta}$ is the corresponding Fourier series. 
The right member in (54) admits a clear interpretation in terms of the celebrated Szegö strong limit theorem (see [6] and the references therein). Indeed, under the assumptions of Corollary 4 ,

$$
h\left(e^{\mathrm{i} \theta}\right)=\frac{f\left(e^{\mathrm{i} \theta}\right)}{g\left(e^{\mathrm{i} \theta}\right)}=\sum_{k=-\infty}^{\infty} h_{k} e^{\mathrm{i} k \theta} \in L^{\infty}(\mathbb{T}),
$$

therefore $h$ naturally generates a Toeplitz operator on the Hardy space $H^{2}(\mathbb{D})$ :

$$
T(h): \phi \rightarrow \mathrm{P}_{+}(h \phi),
$$

where $\phi \in H^{2}(\mathbb{D})$ and $\mathrm{P}_{+}: L^{2}(\mathbb{T}) \rightarrow H^{2}(\mathbb{D})$ is the orthogonal projection. Denote by $t(h)$ the corresponding (infinite) Toeplitz matirx

$$
t(h)_{i j}=h_{i-j}, \quad i, j \geq 1
$$

in the orthonormal basis $\left\{e^{\mathrm{i} k \theta}\right\}_{k>0}$.

Then the Szegö strong limit theorem says that, after an appropriate normalization, the determinants of truncated Toeplitz matrices $\operatorname{det} t_{N}(h)$ (defined by (42)) approach a nonzero limit provided $h$ is sufficiently smooth, has no zeros on $\mathbb{T}$ and the winding number vanishes: $\operatorname{wind}_{\mathbb{T}}(h)=0$ (see [6], $[47])$.

To be more specific, under the assumptions made, the operator $T(1 / h) T(h)$ is of determinant class (see for the definition $[\mathbf{4 7}$, p. 49]) and

$$
\lim _{N \rightarrow \infty} e^{-N(\log h)_{0}} \operatorname{det} t_{N}(h)=\exp \sum_{k=1}^{\infty} k(\log h)_{k}(\log h)_{-k}=\operatorname{det} T(1 / h) T(h),
$$

where $(\log h)_{k}=s_{k}$ are defined by $(52)$. Thus $\mathcal{R}_{\mathbb{D}}(h)=\operatorname{det} T(1 / h) T(h)$.

We have the following determinantal characterization of the resultant (cf. (3)).

Proposition 3. Under assumptions of Corollary 4, the multiplicative commutator

$$
T(g) T(f)^{-1} T(g)^{-1} T(f)
$$

is of determinant class and

$$
\begin{aligned}
\mathcal{R}(f, g) & =\operatorname{det} T\left(\frac{f}{g}\right) T\left(\frac{g}{f}\right)=\operatorname{det}\left[T(f)^{-1} T(g) T(f) T(g)^{-1}\right] \\
& =\lim _{N \rightarrow \infty}\left(\frac{g(0)}{f(\infty)}\right)^{N} \cdot \operatorname{det} t_{N}\left(\frac{f}{g}\right) \\
& =\exp \sum_{k=1}^{\infty} k(\log h)_{k}(\log h)_{-k} .
\end{aligned}
$$

Proof. In view of Corollary 4, it suffices only to establish that the operator determinants and the limit in (56) are equal. Assume that $f$ and $g$ are given by (15). Then

$$
h(z)=\frac{f(z)}{g(z)}=\frac{f(\infty)}{g(0)} \cdot \prod_{i=1}^{m} \frac{1-\frac{a_{i}}{z}}{1-\frac{b_{i}}{z}} \prod_{j=1}^{n} \frac{1-\frac{z}{d_{i}}}{1-\frac{z}{c_{i}}} .
$$


Expanding the logarithm

$$
\log h(z)=\log \frac{f(\infty)}{g(0)}+\sum_{i=1}^{m} \log \frac{1-a_{i} / z}{1-b_{i} / z}+\sum_{j=1}^{n} \log \frac{1-z / d_{j}}{1-z / c_{j}}
$$

in the Laurent series on unit circle $|z|=1$ we obtain: $(\log h)_{0}=\log \frac{f(\infty)}{g(0)}$ and

$$
(\log h)_{k}=\frac{1}{k} \cdot \begin{cases}\sum_{i=1}^{m}\left(a_{i}^{-k}-b_{i}^{-k}\right), & \text { if } \quad k<0 \\ \sum_{j=1}^{n}\left(c_{i}^{-k}-d_{i}^{-k}\right) & \text { if } \quad k>0 .\end{cases}
$$

By the assumptions on the zeros and poles of $f$ and $g$, this yields that $\sum_{k \in \mathbb{Z}}|k| \cdot\left|(\log h)_{k}\right|^{2}<\infty$. By the Widom theorem [56] (see also [47, p. 336]) we conclude that $T(h)^{-1} T(h)-I$ is of trace class. Therefore the Szegö theorem becomes applicable for $h(z)$. Inserting the found value $(\log h)_{0}$ into (55) we obtain

$$
\lim _{N \rightarrow \infty}\left(\frac{g(0)}{f(\infty)}\right)^{N} \cdot \operatorname{det} t_{N}(h)=\operatorname{det} T(1 / h) T(h) .
$$

It remains only to show that

$$
T(1 / h) T(h)=T(f)^{-1} T(g) T(f) T(g)^{-1} .
$$

In order to prove this, notice that by our assumptions $g, 1 / g \in H^{2}(\mathbb{D})$ with $\sup _{z \in \mathbb{D}}|g(z)|<\infty$, and $f(1 / z) \in H^{2}(\mathbb{D})$ with $\inf _{z \in \mathbb{D}}|f(1 / z)|>0$. Thus $h(z)=f(z) / g(z)$ is the Wiener-Hopf factorization (see, for example, [47], Corollary 6.2.3), therefore $T(h)=T(f) T(1 / g)=T(f) T(g)^{-1}$. Similarly we get $T(1 / h)=T(f)^{-1} T(g)$ and desired identity follows.

7.2. Cauchy identity. A related expression for the resultant for two rational functions is given in terms of classical Schur polynomials. Namely, the well-known Cauchy identity [48, p. 299, p. 323] reads as follows:

$$
\prod_{i=1}^{m} \prod_{j=1}^{n} \frac{1}{1-a_{i} c_{j}}=\sum_{\lambda} S_{\lambda}(a) S_{\lambda}(c)=\exp \sum_{k=1}^{\infty} k p_{k}(a) p_{k}(c) .
$$

Here $\lambda=\left(\lambda_{1}, \lambda_{2}, \ldots, \lambda_{k}, \ldots\right)$ denotes a partition, that is a sequence of nonnegative numbers in decreasing order $\lambda_{1} \geq \lambda_{2} \geq \ldots$ with a finite sum,

$$
S_{\lambda}(x) \equiv s_{\lambda}\left(x_{1}, x_{2}, \ldots\right)=\frac{\operatorname{det}\left(x_{i}^{\lambda_{j}+m-j}\right)_{1 \leq i, j \leq m}}{\operatorname{det}\left(x_{i}^{j}\right)_{1 \leq i, j \leq m}}=\frac{\operatorname{det}\left(x_{i}^{\lambda_{j}+m-j}\right)_{1 \leq i, j \leq m}}{\prod_{1 \leq i<j \leq m}\left(x_{i}-x_{j}\right)}
$$

stands for the Schur symmetric polynomials and

$$
p_{k}(a)=\frac{1}{k} \sum_{i=1}^{m} a_{i}^{k}, \quad p_{k}(c)=\frac{1}{k} \sum_{j=1}^{n} c_{j}^{k}
$$

are the so-called power sum symmetric functions.

Note that the series in (57) should be understood in the sense of formal series or the inverse limit (see [33, p. 18]). But if we suppose that

$$
\left|a_{i}\right|<1, \quad\left|c_{j}\right|<1, \quad \forall i, j
$$

then the above identities are valid in the usual sense. 
Let us assume that (58) holds. In order to interpret (57) in terms of the meromorphic resultant, we introduce two rational functions

$$
f(z)=\prod_{i=1}^{m}\left(1-\frac{a_{i}}{z}\right), \quad g(z)=\prod_{j=1}^{n}\left(1-z c_{i}\right) .
$$

We find

$$
\mathcal{R}(f, g)=\frac{\prod_{i=1}^{m} g\left(a_{i}\right)}{g(0)^{m}}=\prod_{i=1}^{m} \prod_{j=1}^{n}\left(1-a_{i} c_{j}\right),
$$

and by comparing with (57) we obtain

$$
\mathcal{R}(f, g)=\exp \left[-\sum_{k=1}^{\infty} k p_{k}(a) p_{k}(c)\right]
$$

By virtue of assumption $(58), \operatorname{supp}(f) \in \mathbb{D}$ and $\operatorname{supp}(g) \in \mathbb{P} \backslash \overline{\mathbb{D}}$, which is consistent with Corollary 4. One can easily see that (59) is a particular case of (54).

\section{Application to the exponential transform of quadrature domains}

8.1. Quadrature domains and the exponential transform. A bounded domain $\Omega$ in the complex plane is called a (classical) quadrature domain $[\mathbf{1}]$, $[\mathbf{4 2}],[\mathbf{4 6}],[\mathbf{2 6}]$ or, in a different terminology, an algebraic domain $[\mathbf{5 3}]$, if there exist finitely many points $z_{i} \in \Omega$ and coefficients $c_{i} \in \mathbb{C}(i=1, \ldots, N$, say) such that

$$
\int_{\Omega} h d x d y=\sum_{i=1}^{N} c_{i} h\left(z_{i}\right)
$$

for every integrable analytic function $h$ in $\Omega$. (Repeated points $z_{i}$ are allowed and should be interpreted as the occurrence of corresponding derivatives of $h$ in the right member.)

An equivalent characterization is due to Aharonov and Shapiro [1] and (under simplifying assumptions) Davis $[\mathbf{1 1}]: \Omega$ is a quadrature domain if and only if there exists a meromorphic function $S(z)$ in $\Omega$ (the poles are located at the quadrature nodes $z_{i}$ ) such that

$$
S(z)=\bar{z} \quad \text { for } z \in \partial \Omega \text {. }
$$

Thus $S(z)$ is the Schwarz function of $\partial \Omega[\mathbf{1 1}],[\mathbf{4 6}]$, which in the above case is meromorphic in all of $\Omega$.

Now let $\Omega$ be an arbitrary bounded open set in the complex plane. The moments of $\Omega$ are the complex numbers:

$$
a_{m n}=\int_{\Omega} z^{m} \bar{z}^{n} d x d y
$$

Recoding this sequence (on the level of formal series) into a new sequence $b_{m n}$ by the rule

$$
\sum_{m, n=0}^{\infty} \frac{b_{m n}}{z^{m+1} \bar{w}^{n+1}}=1-\exp \left(-\sum_{m, n=0}^{\infty} \frac{a_{m n}}{z^{m+1} \bar{w}^{n+1}}\right), \quad|z|,|w| \gg 1,
$$


reveals an established notion of exponential transform $[\mathbf{9}],[\mathbf{3 8}],[\mathbf{2 3}]$. More precisely, this is the function of two complex variables defined by

$$
E_{\Omega}(z, w)=\exp \left[\frac{1}{2 \pi \mathrm{i}} \int_{\Omega} \frac{d \zeta}{\zeta-z} \wedge \frac{d \bar{\zeta}}{\bar{\zeta}-\bar{w}}\right]
$$

It is in principle defined in all $\mathbb{C}^{2}$, but we shall discuss it only in $(\mathbb{C} \backslash \bar{\Omega})^{2}$, where it is analytic/antianalytic.

For large enough $z$ and $w$ we have

$$
E_{\Omega}(z, w)=1-\sum_{m, n=0}^{\infty} \frac{b_{m n}}{z^{m+1} \bar{w}^{n+1}} .
$$

REMARK 7. The exponential transform admits the following operator theoretic interpretation, due to J.D. Pincus [34]. Let $T: H \rightarrow H$ be a bounded linear operator in a Hilbert space $H$, with one rank self-commutator given by

$$
\left[T^{*}, T\right]=T^{*} T-T T^{*}=\xi \otimes \xi,
$$

where $\xi \in H, \xi \neq 0$. Then there is a measurable function $g: \mathbb{C} \rightarrow[0,1]$ with compact support such that

$$
\operatorname{det}\left[T_{z} T_{w}^{*} T_{z}^{-1} T_{w}^{*-1}\right]=\exp \left[\frac{1}{2 \pi \mathrm{i}} \int_{\mathbb{C}} \frac{g(\zeta) d \zeta \wedge d \bar{\zeta}}{(\zeta-z)(\bar{\zeta}-\bar{w})}\right]
$$

where $T_{u}=T-u I$. The function $g$ is called the principal function of $T$. Conversely, for any given function $g$ with values in $[0,1]$ there is an operator $T$ with one rank self-commutator such that (62) holds.

Let $\Omega$ be an arbitrary bounded domain. In [37] M. Putinar proved that the following conditions are equivalent:

a) $\Omega$ is a quadrature domain;

b) $\Omega$ is determined by some finite sequence $\left(a_{m n}\right)_{0 \leq m, n \leq N}$;

c) for some positive integer $N$ there holds

$$
\operatorname{det}\left(b_{m n}\right)_{0 \leq m, n \leq N}=0 ;
$$

d) the function $E_{\Omega}(z, w)$ is rational for $z, w$ large, of the kind

$$
E_{\Omega}(z, w)=\frac{Q(z, w)}{P(z) \overline{P(w)}}
$$

where $P$ and $Q$ are polynomials;

e) there is a bounded linear operator $T$ acting on a Hilbert space $H$, with spectrum equal to $\bar{\Omega}$, with rank one self commutator $\left[T^{*}, T\right]=$ $\xi \otimes \xi(\xi \in H)$ and such that the linear span $\bigvee_{k \geq 0} T^{* k} \xi$ is finite dimensional.

When these conditions hold then the minimum possible number $N$ in b) and c), the degree of $P$ in $\mathbf{d}$ ), and the dimension of $\bigvee_{k>0} T^{* k} \xi$ in e) all coincide with the order of the quadrature domain, i.e., the number $N$ in (60). For $Q$, see more precisely below.

Note that $E_{\Omega}$ is Hermitian symmetric: $E_{\Omega}(w, z)=\overline{E_{\Omega}(z, w)}$ and multiplicative: if $\Omega_{1}$ and $\Omega_{2}$ are disjoint then

$$
E_{\Omega_{1} \cup \Omega_{2}}(z, w)=E_{\Omega_{1}}(z, w) E_{\Omega_{2}}(z, w) .
$$


As $|w| \rightarrow \infty$ one has

$$
E_{\Omega}(z, w)=1-\frac{1}{\bar{w}} K_{\Omega}(z)+\mathcal{O}\left(\frac{1}{|w|^{2}}\right)
$$

with $z \in \mathbb{C}$ fixed, where $K_{\Omega}(z)=\frac{1}{2 \pi \mathrm{i}} \int_{\Omega} \frac{d \zeta \wedge d \bar{\zeta}}{\zeta-z}$ stands for the Cauchy transform of $\Omega$. On the diagonal $w=z$ we have $E_{\Omega}(z, z)>0$ for $z \in \mathbb{C} \backslash \bar{\Omega}$ and

$$
\lim _{z \rightarrow z_{0}} E_{\Omega}(z, z)=0
$$

for almost all $z_{0} \in \partial \Omega$ (see $[\mathbf{2 3}]$ for details). Thus the information of $\partial \Omega$ is explicitly encoded in $E_{\Omega}$.

It is also worth to mention that $1-E_{\Omega}(z, w)$ is positive definite as a kernel, which implies that when $\Omega$ is a quadrature domain of order $N$ then $Q(z, w)$ admits the following representation $[\mathbf{2 4}]$ :

$$
Q(z, w)=P(z) \overline{P(w)}-\sum_{k=0}^{N-1} P_{k}(z) \overline{P_{k}(w)},
$$

where $\operatorname{deg} P_{k}=k$.

In the simplest case, when $\Omega=\mathbb{D}(0, r)$, the disk centered at the origin and of radius $r$, the Cauchy transform and the Schwarz function coincide and are equal to $\frac{r^{2}}{z}$, and

$$
E_{\mathbb{D}(0, r)}(z, w)=1-\frac{r^{2}}{z \bar{w}} .
$$

8.2. The elimination function on a Schottky double. Let $\Omega$ be a finitely connected plane domain with analytic boundary or, more generally, a bordered Riemann surface and let

$$
M=\widehat{\Omega}=\Omega \cup \partial \Omega \cup \widetilde{\Omega}
$$

be the Schottky double of $\Omega$, i.e., the compact Riemann surface obtained by completing $\Omega$ with a backside with the opposite conformal structure, the two surfaces glued together along $\partial \Omega$ (see [14], for example). On $\widehat{\Omega}$ there is a natural anticonformal involution $\phi: \widehat{\Omega} \rightarrow \widehat{\Omega}$ exchanging corresponding points on $\Omega$ and $\widetilde{\Omega}$ and having $\partial \Omega$ as fixed points.

Let $f$ and $g$ be two meromorphic functions on $\widehat{\Omega}$. Then

$$
\left.\left.f^{*}=\overline{(f \circ \phi}\right), \quad g^{*}=\overline{(g \circ \phi}\right) .
$$

are also meromorphic on $\widehat{\Omega}$.

Theorem 6. With $\Omega, \widehat{\Omega}, f, g$ as above, assume in addition that $f$ has no poles in $\Omega \cup \partial \Omega$ and that $g$ has no poles in $\widetilde{\Omega} \cup \partial \Omega$. Then, for large $z, w$,

$$
\mathcal{E}_{f, g}(z, \bar{w})=\exp \left[\frac{1}{2 \pi \mathrm{i}} \int_{\Omega} \frac{d f}{f-z} \wedge \frac{d \overline{g^{*}}}{\overline{g^{*}}-\bar{w}}\right] .
$$

In particular,

$$
\mathcal{E}_{f, f^{*}}(z, \bar{w})=\exp \left[\frac{1}{2 \pi \mathrm{i}} \int_{\Omega} \frac{d f}{f-z} \wedge \frac{d \bar{f}}{\bar{f}-\bar{w}}\right]
$$


Proof. For the divisors of $f-z$ and $g-w$ we have, if $z, w$ are large enough, $\operatorname{supp}(f-z) \subset \widetilde{\Omega}, \operatorname{supp}(g-w) \subset \Omega$. Moreover, $\log (g-w)$ has a single-valued branch in $\widetilde{\Omega}$ (because the image $g(\widetilde{\Omega})$ is contained in some disk $\mathbb{D}(0, R)$, hence $(g-w)(\widetilde{\Omega})$ is contained in $\mathbb{D}(-w, R)$, hence $\log (g-w)$ can be chosen single-valued in $\widetilde{\Omega}$ if $|w|>R$ ). Using that $g=\overline{g^{*}}$ on $\partial \Omega$ we therefore get

$$
\begin{aligned}
\mathcal{E}_{f, g}(z, \bar{w}) & =\exp \left[\frac{1}{2 \pi \mathrm{i}} \int_{\hat{\Omega}} \frac{d f}{f-z} \wedge d \log (g-\bar{w})\right]=\exp \left[\frac{1}{2 \pi \mathrm{i}} \int_{\Omega} \frac{d f}{f-z} \wedge d \log (g-\bar{w})\right] \\
& =\exp \left[-\frac{1}{2 \pi \mathrm{i}} \int_{\partial \Omega} \frac{d f}{f-z} \wedge \log (g-\bar{w})\right]=\exp \left[-\frac{1}{2 \pi \mathrm{i}} \int_{\partial \Omega} \frac{d f}{f-z} \wedge \log \left(\overline{g^{*}}-\bar{w}\right)\right] \\
& =\exp \left[\frac{1}{2 \pi \mathrm{i}} \int_{\Omega} \frac{d f}{f-z} \wedge \frac{d g^{*}}{g^{*}-\bar{w}}\right] .
\end{aligned}
$$

as claimed.

\subsection{The exponential transform as the meromorphic resultant.} Let $S(z)$ be the Schwarz function of a quadrature domain $\Omega$. Then the relation (61) can be interpreted as saying that the pair of functions $S(z)$ and $\bar{z}$ on $\Omega$ combines into a meromorphic function on the Schottky double $\widehat{\Omega}=\Omega \cup \partial \Omega \cup \widetilde{\Omega}$ of $\Omega$, namely the function $g$ which equals $S(z)$ on $\Omega, \bar{z}$ on $\widetilde{\Omega}$.

The function $f=g^{*}=\overline{g \circ \phi}$ is then represented by the opposite pair: $z$ on $\Omega, \overline{S(z)}$ on $\widetilde{\Omega}$. It is known $[\mathbf{2 2}]$ that $f$ and $g=f^{*}$ generate the field of meromorphic functions on $\widehat{\Omega}$, and we call this pair the canonical representation of $\Omega$ in $\widehat{\Omega}$

From Theorem 6 we immediately get

THEOREM 7. For any quadrature domain $\Omega$

$$
E_{\Omega}(z, w)=\mathcal{E}_{f, f^{*}}(z, \bar{w}) \quad(|z|,|w| \gg 1),
$$

where $f, f^{*}$ is the canonical representation of $\Omega$ in $\widehat{\Omega}$.

Here we used Theorem 6 with $f(\zeta)=\zeta$ on $\Omega$, i.e., $\left.f\right|_{\Omega}=$ id. A slightly more flexible way of formulating the same result is to let $f$ be defined on an independent surface $W$, so that $f: W \rightarrow \Omega$ is a conformal map. Then $\Omega$ is a quadrature domain if and only if $f$ extends to a meromorphic function of the Schottky double $\widehat{W}$ (this is an easy consequence of (61); cf. [22]). When this is the case the exponential transform of $\Omega$ is

$$
E_{\Omega}(z, w)=\mathcal{E}_{f, f^{*}}(z, \bar{w})
$$

with the elimination function in the right member now taken in $\widehat{W}$.

REMARK 8. If $\Omega$ is simply connected one may take $W=\mathbb{D}$, so that $\widehat{W}=\mathbb{P}$ with involution $\phi: \zeta \mapsto 1 / \bar{\zeta}$. Then $f: \mathbb{D} \rightarrow \Omega$ is a rational function when (and only when) $\Omega$ is a quadrature domain, hence we conclude that $E_{\Omega}(z, w)$ in this case is the elimination function for two rational functions, $f(\zeta)$ and $\left.f^{*}(\zeta)=\overline{f(1 / \bar{\zeta}}\right)$. This topic will be pursued in Section 8.5. 
In analogy with (27) one can also introduce an extended version of the exponential transform:

$$
E_{\Omega}\left(z, w ; z_{0}, w_{0}\right):=\exp \left[\frac{1}{2 \pi \mathrm{i}} \int_{\Omega}\left(\frac{d \zeta}{\zeta-z}-\frac{d \zeta}{\zeta-z_{0}}\right) \wedge\left(\frac{d \bar{\zeta}}{\bar{\zeta}-\bar{w}}-\frac{d \bar{\zeta}}{\bar{\zeta}-\bar{w}_{0}}\right)\right] .
$$

One advantage with this extended exponential transform is that it is defined for a wider class of domains, for example, for the entire complex plane. If the standard exponential transform is well-defined then

$$
E_{\Omega}\left(z, w ; z_{0}, w_{0}\right)=\frac{E_{\Omega}(z, w) E_{\Omega}\left(z_{0}, w_{0}\right)}{E_{\Omega}\left(z, w_{0}\right) E_{\Omega}\left(z_{0}, w\right)} .
$$

In other direction, the standard exponential transform can be obtained from the extended version by passing to the limit:

$$
E_{\Omega}(z, w)=\lim _{z_{0}, w_{0} \rightarrow \infty} E_{\Omega}\left(z, w ; z_{0}, w_{0}\right) .
$$

Arguing as in the proof of Theorem 7 we obtain the following generalization.

COROLlary 5. Let $\Omega$ is a quadrature domain with canonical representation $f$ and $f^{*}$. Then

$$
E_{\Omega}\left(z, w ; z_{0}, w_{0}\right)=\mathcal{E}_{f, f^{*}}\left(z, \bar{w} ; z_{0}, \bar{w}_{0}\right),
$$

where $\mathcal{E}_{f, f^{*}}\left(z, w ; z_{0}, w_{0}\right)$ is the extended elimination function (27).

8.4. Rational maps. Now we study how the exponential transform of an arbitrary domain in $M=\mathbb{P}$ behaves under rational maps. For simplicity, we only deal with bounded domains, but this restriction is not essential. It can be easily removed by passing to the extended version of the exponential transform.

For domains in general, the exponential transform need not be rational. However we still have the limit relation (65). This makes it possible to continue $E_{\Omega}$ at infinity by

$$
E_{\Omega}(z, \infty)=E_{\Omega}(\infty, w)=E_{\Omega}(\infty, \infty)=1 .
$$

TheOREM 8. Let $\Omega_{i}, i=1,2$, be two bounded open sets in the complex plane and $F$ be a p-valent proper rational function which maps $\Omega_{1}$ onto $\Omega_{2}$. Then for all $z, w \in \mathbb{C} \backslash \bar{\Omega}_{2}$

$$
E_{2}^{p}(z, w)=E_{1}((F-z),(F-w))=\mathcal{R}_{u}\left(F(u)-z, \mathcal{R}_{v}\left(F(v)-w, E_{1}(u, v)\right)\right),
$$

where $E_{k}=E_{\Omega_{k}}$. (See (21) for the notation.)

Proof. We have

$$
E_{2}^{p}(z, w)=\exp \left(\frac{p}{2 \pi \mathrm{i}} \int_{\Omega_{2}} \frac{d \zeta \wedge d \bar{\zeta}}{(\zeta-z)(\bar{\zeta}-\bar{w})}\right)=\exp \left(\frac{1}{2 \pi \mathrm{i}} \int_{\Omega_{1}} \frac{F^{\prime}(\zeta) \overline{F^{\prime}(\zeta)} d \zeta \wedge d \bar{\zeta}}{(F(\zeta)-z)(\overline{F(\zeta)}-\bar{w})}\right) .
$$

Let $D_{u}$ denote the divisor of $F(\zeta)-u$. Then

$$
\frac{F^{\prime}(\zeta)}{F(\zeta)-z}=\frac{d}{d \zeta} \log (F(\zeta)-z)=\sum_{\alpha \in \mathbb{P}} \frac{D_{z}(\alpha)}{\zeta-\alpha}
$$


where the latter sum is finite. Conjugating both sides in this identity for $z=w$ we get

$$
\frac{\overline{F^{\prime}(\zeta)}}{\overline{F(\zeta)}-\bar{w}}=\sum_{\beta \in \mathbb{P}} \frac{D_{w}(\beta)}{\bar{\zeta}-\bar{\beta}}
$$

therefore,

$$
\frac{F^{\prime}(\zeta) \overline{F^{\prime}(\zeta)}}{(F(\zeta)-z)(\overline{F(\zeta)}-\bar{w})}=\sum_{\alpha \in \mathbb{P}} \sum_{\beta \in \mathbb{P}} \frac{D_{z}(\alpha) D_{w}(\beta)}{(\zeta-\alpha)(\bar{\zeta}-\bar{\beta})}
$$

By assumptions, $F(\zeta)-u$ is different from 0 and $\infty$ for any choice of $u \in \mathbb{C} \backslash \bar{\Omega}_{2}$ and $\zeta \in \bar{\Omega}_{1}$. Hence $\operatorname{supp} D_{u} \subset \mathbb{C} \backslash \bar{\Omega}_{1}$. Thus successively taking the integral over $\Omega_{1}$ and the exponential gives

$$
E_{2}^{p}(z, w)=\prod_{\alpha, \beta \in \mathbb{P}} E_{1}(\alpha, \beta)^{D_{z}(\alpha) D_{w}(\beta)}=E_{1}\left(D_{z}, D_{w}\right),
$$

which is the first equality in (67). Applying (26) we get the second equality.

Since the exponential transform is a hermitian symmetric function of its arguments, a certain care is needed when using formula (67). The lemma below shows that the meromorphic resultant is merely Hermitian symmetric when one argument is anti-holomorphic. Indeed, suppose, for example, that $f$ is holomorphic and $g$ is anti-holomorphic, that is $g(z)=\overline{h(z)}$, where $h$ is a holomorphic function. Note that $(g)=(h)$. Therefore

$$
\mathcal{R}(g, f)=f((g))=f((h))=h((f))=\overline{g((f)})=\overline{\mathcal{R}(f, g)} .
$$

In summary we have

LEMMA 4. Let $f(z)$ be holomorphic (or anti-holomorphic) and $g(z)$ be anti-holomorphic (holomorphic resp.) in z. Then

$$
\mathcal{R}(g, f)=\overline{\mathcal{R}(f, g)} .
$$

COROLlary 6. Under the conditions of Theorem 8, if $E_{1}$ is rational then $E_{2}^{p}$ is also rational.

Proof. First consider the inner resultant $\mathcal{R}_{v}(\cdot, \cdot)$ in $(67)$. Since $E_{1}(u, v)$ and $F(v)-w$ are rational and $E_{1}$ is hermitian, the resultant is a rational function in $u$ and $\bar{w}$ by virtue of (29) and Sylvester's representation (12) (see also Lemma 4). Repeating this for $\mathcal{R}_{u}(\cdot, \cdot)$ we get the desired property.

REMARK 9. The fact that rationality of the exponential transform is invariant under the action of rational maps is not essentially new. In the separable case, that is when $E_{\Omega_{1}}$ is given by a formula like (63), and in addition $f$ is a one-to-one mapping, the rationality of $E_{\Omega_{2}}$ was proven by M. Putinar (see Theorem 4.1 in [37]). This original proof used existence of the principal function (see Remark 7). 
8.5. Simply connected quadrature domains. Even for quadrature domains, Theorem 8 provides a new effective tool for computing the exponential transform and, thereby, gives explicit information about the complex moments, the Schwarz function etc.

Suppose that $\Omega$ is a simply connected bounded domain and $F$ is a uniformizing map from the unit disk $\mathbb{D}$ onto $\Omega$. P. Davis $[\mathbf{1 1}]$ and D. Aharonov and H.S. Shapiro $[\mathbf{1}]$ proved that $\Omega$ is a quadrature domain if and only if $F$ is a rational function. The we have (cf. Remark 8).

THEOREM 9. Let $F$ be a univalent rational map of the unit disk onto a bounded domain $\Omega$. Then

$$
E_{\Omega}(z, w)=\mathcal{R}_{u}\left(F(u)-z, F^{*}(u)-\bar{w}\right)
$$

where $F^{*}(u)=\overline{F\left(\frac{1}{\bar{u}}\right)}$.

Proof. We have from (66) that $E_{\mathbb{D}}(u, v)=1-\frac{1}{u \bar{v}}$. Hence $E_{\mathbb{D}}(u, \cdot)$ has a zero at $\frac{1}{\bar{u}}$ and a pole at the origin, both of order one. Applying (68) we find

$\left.\mathcal{R}_{v}\left(F(v)-w, E_{\mathbb{D}}(u, v)\right)=\overline{\mathcal{R}_{v}\left(E_{\mathbb{D}}(u, v), F(v)-w\right.}\right)=\frac{\overline{F\left(\frac{1}{\bar{u}}\right)}-\bar{w}}{\overline{F(0)}-\bar{w}}=\frac{F^{*}(u)-\bar{w}}{\overline{F(0})-\bar{w}}$.

Taking into account the null-homogeneity (24) of resultant and using Theorem 8 we obtain (69).

Applying (29) can we write the resultant in the right hand side of (69) explicitly.

Corollary 7. Let $F(\zeta)=\frac{A(\zeta)}{B(\zeta)}$ be a univalent rational map of the unit disk onto a bounded domain $\Omega$, where $B$ is normalized to be a monic polynomial. Then

$$
E_{\Omega}(z, w)=\mathcal{R}_{\mathrm{pol}}\left(B, B^{\sharp}\right) \cdot \frac{\mathcal{R}_{\mathrm{pol}}\left(P_{z}, P_{w}^{\sharp}\right)}{T(z) \overline{T(w)}},
$$

where $m=\operatorname{deg} B, n=\max (\operatorname{deg} A, \operatorname{deg} B)=\operatorname{deg} F, P_{t}=A-t B$,

$$
T(z)=(F(0)-z)^{n-m} \mathcal{R}_{\mathrm{pol}}\left(P_{z}, B^{\sharp}\right),
$$

and $P^{\sharp}(\zeta)=\zeta^{\operatorname{deg} P} \overline{P(1 / \bar{\zeta})}$ is the so-called reciprocal polynomial.

We finish this section by demonstrating some concrete examples. First we apply the above results to polynomial domains. Let, in Corollary 7 , $F(\zeta)=a_{1} \zeta+\ldots+a_{n} \zeta^{n}$ be a polynomial. Then $B=B^{\sharp} \equiv 1, T(z)=z^{n}$ and

$$
P_{z}(\zeta)=-z+a_{1} \zeta+\ldots+a_{n} \zeta^{n}, \quad P_{w}^{\sharp}(\zeta)=\bar{a}_{n}+\ldots+\bar{a}_{1} \zeta^{n-1}-\bar{w} \zeta^{n} .
$$


This gives the following closed formula.

$$
E_{\Omega}(z, w)=\operatorname{det}\left(\begin{array}{cccccc}
-1 & & & \frac{\bar{a}_{n}}{\bar{w}} & & \\
\frac{a_{1}}{z} & \ddots & & \vdots & \ddots & \\
\vdots & & -1 & \frac{\bar{a}_{1}}{\bar{w}} & & \frac{\bar{a}_{1}}{\bar{w}} \\
\frac{a_{n}}{z} & & \frac{a_{1}}{z} & -1 & & \vdots \\
& \ddots & \vdots & & \ddots & \frac{\bar{a}_{1}}{\bar{w}} \\
& & \frac{a_{n}}{z} & & & -1
\end{array}\right) .
$$

A similar determinantal representation is valid also for general rational functions $F$.

For $n=1$ and $n=2,(71)$ becomes

$$
\begin{aligned}
& E_{\Omega}(z, w)=1-x_{1} y_{1}, \\
& E_{\Omega}(z, w)=1-x_{1} y_{1}-2 x_{2} y_{2}-x_{2}^{2} y_{2}^{2}-x_{1} x_{2} y_{1} y_{2}+x_{1}^{2} y_{2}+x_{2} y_{1}^{2},
\end{aligned}
$$

where $x_{i}=a_{i} / z$ and $y_{i}=\bar{a}_{i} / \bar{w}$.

The determinant in (71), and, more generally, the resultant in (69), has the following transparent interpretation in terms of the Schwarz function. Suppose that $\Omega=F(\mathbb{D})$ for a rational function $F$ and recall the definition (61) of the Schwarz function of $\partial \Omega: S(z)=\bar{z}, z \in \partial \Omega$. After substitution $z=F(\zeta),|\zeta|=1$, this yields

$$
S(F(\zeta))=\overline{F(\zeta)}=\bar{F}\left(\frac{1}{\zeta}\right)=F^{*}(\zeta)
$$

Note that $F^{*}(\zeta)$ is a rational function again. Thus the Schwarz function may be found by elimination of the variable $\zeta$ in the following system of rational equations:

$$
\left\{\begin{array}{c}
w=F^{*}(\zeta), \\
z=F(\zeta),
\end{array}\right.
$$

where $w=S(z)$. Namely, by Proposition 1 the system (72) holds for some $\zeta$ if and only if

$$
\mathcal{R}_{\zeta}\left(F(\zeta)-z, F^{*}(\zeta)-w\right)=0
$$

The latter provides an implicit equation for $w=S(z)$ in terms of $z$. Note that the expression on the left hand side in (73) is exactly the exponential transform $E_{\Omega}(z, \bar{w})$ in (69). In fact, Theorem 7 implies that for any quadrature domain $\Omega$ one has $E_{\Omega}(z, \overline{S(z)})=0$.

\section{Meromorphic resultant versus polynomial}

Recall that the meromorphic resultant vanishes identically for polynomials (considered as meromorphic functions on $\mathbb{P}$ ). This makes it natural to ask whether there is any reasonable reduction of the meromorphic resultant to the polynomial one. Here we shall discuss this question and show how to adapt the main definitions to make them sensible in the polynomial case.

First we recall the concept of local symbol (see, for example, [45], [51]). Let $f, g$ be meromorphic functions on an arbitrary Riemann surface $M$. 
Notice that for any $a \in M$, the limit

$$
\tau_{a}(f, g):=(-1)^{\operatorname{ord}_{a} f \operatorname{ord}_{a} g} \lim _{z \rightarrow a} \frac{f(z)^{\operatorname{ord}_{a} g}}{g(z)^{\operatorname{ord}_{a} f}}
$$

exists and it is a nonzero complex number. This number is called the local symbol of $f, g$ at $a$.

For all but finitely many $a$ we have $\tau_{a}(f, g)=1$. The following properties follow from the definition:

$$
\tau_{a}(f, g) \tau_{a}(g, f)=1
$$

multiplicativity

$$
\tau_{a}(f, g) \tau_{a}(f, h)=\tau_{a}(f, g h)
$$

and

$$
\tau_{a}(f, g)^{\operatorname{ord}_{a} h} \tau_{a}(g, h)^{\operatorname{ord}_{a} f} \tau_{a}(h, f)^{\operatorname{ord}_{a} g}=(-1)^{\operatorname{ord}_{a} f \cdot \operatorname{ord}_{a} g \cdot \operatorname{ord}_{a} h} .
$$

In this notation, Weil's reciprocity law in its full strength states that on a compact $M$, the product of the local symbols of any two meromorphic functions $f$ and $g$ equals one:

$$
\prod_{a \in M} \tau_{a}(f, g)=1
$$

Definition 4. Let $a \in M$ and let $f$ and $g$ be two meromorphic functions which are admissible on $M \backslash\{a\}$. Let $\sigma=\sigma(\zeta)$ be a local coordinate at $a$ normalized such that $\sigma(a)=0$. Then the following product is well-defined:

$$
\mathcal{R}_{\sigma}(f, g)=\frac{\tau_{a}(\sigma, g)^{\operatorname{ord}_{a} f}}{\tau_{a}(f, g)} \prod_{\xi \neq a} g(\xi)^{\operatorname{ord}_{\xi} f}
$$

and is called the reduced (with respect to $\sigma$ ) resultant.

Proposition 4. Under the above assumptions,

$$
\mathcal{R}_{\sigma}(f, g)=(-1)^{\operatorname{ord}_{a} f \operatorname{ord}_{a} g} \cdot \mathcal{R}_{\sigma}(g, f),
$$

and

$$
\mathcal{R}_{\sigma}\left(f_{1} f_{2}, g\right)=\mathcal{R}_{\sigma}\left(f_{1}, g\right) \mathcal{R}_{\sigma}\left(f_{2}, g\right) .
$$

Moreover, if $\sigma^{\prime}$ is another local coordinate with $\sigma^{\prime}(a)=0$, then

$$
\mathcal{R}_{\sigma^{\prime}}(f, g)=\left(-\tau_{\xi}\left(\sigma^{\prime}, \sigma\right)\right)^{\operatorname{ord}_{a} f \operatorname{ord}_{a} g} \mathcal{R}_{\sigma}(f, g) .
$$

Proof. Note first $\mathcal{R}_{\sigma}(f, g)$ vanishes or equals infinity if and only if $\mathcal{R}_{\sigma}(g, f)$ does so. Indeed, let us assume that, for instance, $\mathcal{R}_{\sigma}(f, g)=0$. Then it follows from $(78)$ and the fact that $\tau_{a}(\cdot, \cdot)$ is finite and never vanishes, that $g\left(\xi_{0}\right)^{\operatorname{ord}_{\xi_{0}}(f)}=0$ for some $\xi_{0} \neq a$. Hence $\operatorname{ord}_{\xi_{0}}(f) \operatorname{ord}_{\xi_{0}}(g)>0$, and $f\left(\xi_{0}\right)^{\operatorname{ord}_{\xi_{0}}(g)}=0$. From the admissibility condition we know that the product $\operatorname{ord}_{\xi}(f) \operatorname{ord}_{\xi}(g)$ does not change sign on $M \backslash\{a\}$, therefore $\operatorname{ord}_{\xi}(f) \operatorname{ord}_{\xi}(g) \geq$ 0 everywhere. Then changing roles of $f$ and $g$ in $(78)$, we get $\mathcal{R}_{\sigma}(g, f)=0$.

Thus without loss of generality we may assume that $\mathcal{R}_{\sigma}(f, g) \neq 0$ and $\mathcal{R}_{\sigma}(f, g) \neq \infty$. By virtue of the definition of admissibility we see that the product $\operatorname{ord}_{\xi} f \operatorname{ord}_{\xi} g$ is semi-definite on $M \backslash\{a\}$, hence

$$
\operatorname{ord}_{\xi} f \operatorname{ord}_{\xi} g=0 \quad(\xi \in M \backslash\{a\}) \text {. }
$$


Since $\operatorname{ord}_{a} \sigma=1$, we have by (76) and (74)

$$
\frac{\tau_{a}(\sigma, f)^{\operatorname{ord}_{a} g}}{\tau_{a}(\sigma, g)^{\operatorname{ord}_{a} f}}=\tau_{a}(g, \sigma)^{\operatorname{ord}_{a} f} \tau_{a}(\sigma, f)^{\operatorname{ord}_{a} g}=(-1)^{\operatorname{ord}_{a} f \operatorname{ord}_{a} g} \tau_{a}(g, f)
$$

We have

$$
\begin{aligned}
\frac{\mathcal{R}_{\sigma}(g, f)}{\mathcal{R}_{\sigma}(f, g)} & =\frac{\tau_{a}(f, g) \tau_{a}(\sigma, f)^{\operatorname{ord}_{a} g}}{\tau_{a}(g, f) \tau_{a}(\sigma, g)^{\operatorname{ord}_{a} f}} \prod_{\xi \neq a} \frac{f(\xi)^{\operatorname{ord}_{\xi}(g)}}{g(\xi)^{\operatorname{ord}_{\xi}(f)}} \\
& =(-1)^{\operatorname{ord}_{a} f \operatorname{ord}_{a} g} \tau_{a}(f, g) \prod_{\xi \neq a} \frac{f(\xi)^{\operatorname{ord}_{\xi}(g)}}{g(\xi)^{\operatorname{ord}_{\xi}(f)}} \\
& =(-1)^{\operatorname{ord}_{a} f \operatorname{ord}_{a} g} \tau_{a}(f, g) \prod_{\xi \neq a}(-1)^{\operatorname{ord}_{\xi} f \operatorname{ord}_{\xi} g} \tau_{\xi}(f, g)
\end{aligned}
$$

Hence, by virtue of (82) and (77) we obtain

$$
\frac{\mathcal{R}_{\sigma}(g, f)}{\mathcal{R}_{\sigma}(f, g)}=(-1)^{\operatorname{ord}_{a} f \operatorname{ord}_{a} g} \prod_{\xi \in M} \tau_{\xi}(f, g)=(-1)^{\operatorname{ord}_{a} f \operatorname{ord}_{a} g},
$$

and (79) follows.

In order to prove (80), it suffices to notice that the right side of $(78)$ is multiplicative, by virtue of $(75)$, with respect to $f$.

Finally, we notice that by (76): $\tau_{a}\left(\sigma^{\prime}, g\right) \tau_{a}(g, \sigma) \tau_{a}\left(\sigma, \sigma^{\prime}\right)^{\operatorname{ord}_{a} g}=(-1)^{\operatorname{ord}_{a} g}$, hence

$$
\frac{\mathcal{R}_{\sigma^{\prime}}(f, g)}{\mathcal{R}_{\sigma}(f, g)}=\left(\frac{\tau_{a}\left(\sigma^{\prime}, g\right)}{\tau_{a}(\sigma, g)}\right)^{\operatorname{ord}_{a} f}=\left(-\tau_{a}\left(\sigma^{\prime}, \sigma\right)\right)^{\operatorname{ord}_{a} g \operatorname{ord}_{a} f}
$$

and the required formula (81) follows.

Now we apply some of the above constructions to the polynomial case. On the Riemann sphere, $\mathbb{P}$, we pick the distinguished point $a=\infty$ and the corresponding local coordinate $\sigma(z)=\frac{1}{z}$. Since any two polynomials form an admissible pair on $\mathbb{C}$, the corresponding product in (78) is well-defined.

Let us consider two arbitrary polynomials $f$ and $g$. Since $\operatorname{ord}_{\xi} f \cdot \operatorname{ord}_{\xi} g \geq$ 0 for any point $\xi$, we see that $\mathcal{R}_{\sigma}(f, g)=0$ if and only if $f$ and $g$ have a common zero in $\mathbb{C}$. In particular, $\mathcal{R}_{\sigma}(f, g) \neq 0$ for coprime polynomials.

Now let $f$ and $g$ have no common zeros. In the notation of (9) we have $\operatorname{ord}_{\infty} g=-n$ and

$$
\tau_{\infty}(\sigma, g)=(-1)^{n} \lim _{z \rightarrow \infty} \frac{z^{\operatorname{deg} g}}{g(z)}=\frac{(-1)^{n}}{g_{n}}
$$

and

hence

$$
\tau_{\infty}(f, g)=(-1)^{n m} \lim _{z \rightarrow \infty} \frac{f(z)^{-n}}{g(z)^{-m}}=(-1)^{n m} \frac{g_{n}^{m}}{f_{m}^{n}}
$$

$$
\mathcal{R}_{\sigma}(f, g)=f_{m}^{n} \prod_{\xi \neq \infty} g(\xi)^{\operatorname{ord}_{\xi}(f)}=f_{m}^{n} g_{n}^{m} \prod_{i=1}^{m} \prod_{j=1}^{n}\left(a_{i}-c_{j}\right)
$$

Thus, comparing this with (10), we recover the classical definition of polynomial resultant. We have therefore proved the following. 
Corollary 8. Let $M=\mathbb{P}$ and $\sigma(z)=\frac{1}{z}$ be the standard local coordinate at $\infty$. Then

$$
\mathcal{R}_{\sigma}(f, g)=\mathcal{R}_{\text {pol }}(f, g) .
$$

A beautiful interpretation of the product in the right hand side of (78) as a determinant is given in a recent paper of J.-L. Brylinski and E. Previato [5]. In particular, the authors show that this product is described as the determinant $\operatorname{det}(f, A / g A)$ of the Koszul double complex for $f$ and $g$ acting on $A=H^{0}(M \backslash\{a\}, \mathcal{O})$.

\section{References}

[1] D. Aharonov, H. S. Shapiro, Domains in which analytic functions satisfy quadrature identities, J. Analyse Math. 30 (1976), 39-73.

[2] E.L. Basor, P.J. Forrester, Formulas for the evaluation of Toeplitz determinants with rational generating functions, Math. Nachr. 170 (1994), 5-18.

[3] G. Baxter, P. Schmidt, Determinants of a certain class of non-hermitian Toeplitz matrices, Math. Scand., 9 (1961), 122-128.

[4] P. Bikker, A.Yu. Uteshev, On the Bezout construction of the resultant. Polynomial elimination-algorithms and applications. J. Symbolic Comput. 28(1999), no. 1-2, 45-88.

[5] J.-L. Brylinski, E. Previato, Koszul Complexes, Differential Operators, and the WeilTate Reciprocity Law. J. of Algebra, 230 (2000), 89-100.

[6] A. Böttcher, B. Silbermann, Introduction to large truncated Toeplitz matrices. Universitext. Springer-Verlag, New York, 1999.

[7] F. Calogero, Remarkable matrices and trigonometric identities, J. Comput. Appl. Math. 83(1997), no. 1, 127-130.

[8] F. Calogero, Remarkable Matrices and Trigonometric Identities. II., Commun. Appl. Math. 3(1999), 267-270.

[9] R. W. Carey, J. D. Pincus, An exponential formula for determining functions, Indiana Univ. Math.J. 23 (1974), 1031-1042.

[10] E. Cattani, A. Dickenstein and B. Sturmfels, Residues and resultants, J. Math. Sci. Univ. Tokyo 5 (1998), 119-148.

[11] P. J. Davis, The Schwarz Function and its Applications, Carus Math. Mongraphs No. 17, Math. Assoc. Amer., 1974.

[12] K. M. Day, Toeplitz matrices generated by the Laurent series expansion of an arbitrary rational function., Trans. Amer. Math. Soc. 206(1975), 224-245.

[13] P. Deligne, Le symbole modéré, Inst. Hautes Études Sci. Publ. Math. No. 73 (1991), $147-181$.

[14] H. Farkas, I. Kra, Riemann surfaces, Graduate Texts in Mathematics, 71. SpringerVerlag, New York-Berlin, 1980.

[15] M.E. Fisher, R.E. Hartwig, Asymptotic behavior of Toeplitz matrices and determinants. Arch. Rational Mech. Anal. 32 (1969), 190-225.

[16] O. Forster, Riemannsche Flächen, Springer-Verlag, 1977.

[17] P.J. Forrester, A constant term identity and its relationship to the log-gas and some quantum many body systems, Physics Letters A, 163 (1992), issue 1-2, p. 121-126.

[18] W. Fulton, P. Pragacz, Schubert varieties and degeneracy loci. Lect. Notes Math., 1689. Springer-Verlag, Berlin, 1998.

[19] I.M. Gelfand, M.M. Kapranov, A.V. Zelevinsky, Discriminants, resultants, and multidimensional determinants. Birkhäuser Boston, Inc., Boston, MA, 1994.

[20] P. Griffith, J. Harris, Principles of Algebraic Geometry, Wiley \& Sons, 1978.

[21] R.C. Gunning, Lectures on Riemann Surfaces - Jacobi Varieties, Princeton Mathematical Texts, Princeton, 1972.

[22] B. Gustafsson, Quadrature identities and the Schottky double, Acta Appl. Math. 1 (1983), 209-240.

[23] B. Gustafsson, M. Putinar, An exponential transform and regularity of free boundaries in two dimensions, Ann. Scuola Norm. Sup. Pisa Cl. Sci (4), 26 (1998), 507-543. 
[24] B. Gustafsson, M. Putinar, Linear analysis of quadrature domains, II, Israel J. Math. 119 (2000), 187-216.

[25] B. Gustafsson, M. Putinar, Selected topics on quadrature domains, Physica D, 235 (2007), 90-100.

[26] B. Gustafsson, H.S. Shapiro, What is a quadrature domain? pp. 1-25 in [39].

[27] B. Gustafsson, V. Tkachev, Resultant identities, in preparation.

[28] R. E. Hartwig, Toeplitz determinants and Szego's formula. J. Austral. Math. Soc. 9 (1969), 62-99.

[29] A. Kasman, E. Previato, Commutative partial differential operators, Physica D, 152153 (2001), 66-77.

[30] I.K. Kostov, I. Krichever, M. Mineev-Weinstein, P.B. Wiegmann, A. Zabrodin, The $\tau$-function for analytic curves. in Random matrix models and their applications, 285299, Math. Sci. Res. Inst. Publ., 40, Cambridge Univ. Press, Cambridge, 2001.

[31] I.M. Krichever, Integration of nonlinear equations by methods of algebraic geometry, Funkts. Anal. Prilozh., 11(1977), no. 1, 15-31.

[32] A. Lascoux, P. Pragacz, Double Sylvester sums for subresultants and multi-Schur functions, J. Symb. Comp., 35(2003), 689-710.

[33] I. D. Macdonald, Symmetric functions and Hall polynomials, 2nd Ed., Oxford Math. Monographs, 1995.

[34] J. D. Pincus, Commutators and systems of singular integral equations, I, Acta Math. 121 (1968), 219-249.

[35] E. Previato, Another algebraic proof of Weil's reciprocity. Atti Accad. Naz. Lincei Cl. Sci. Fis. Mat. Natur. Rend. Lincei (9) Mat. Appl. 2 (1991), no. 2, 167-171.

[36] E. Previato, Seventy years of spectral curves: 1923-1993. Integrable systems and quantum groups (Montecatini Terme, 1993), 419-481, Lecture Notes in Math., 1620, Springer, Berlin, 1996.

[37] M. Putinar, Extremal solutions of the two-dimensional L-problem of moments, J.Funct.An. 136 (1996), 331-364.

[38] M. Putinar, Extremal solutions of the two-dimensional L-problem of moments, II, J. Approx. Th. 92 (1998), 38-58.

[39] Quadrature Domains and Applications, a Harold S. Shapiro Anniversary Volume. (eds. P. Ebenfelt, B. Gustafsson, D. Khavinson, M. Putinar), Birkhäuser, 2005.

[40] Yu. Rodin, The Riemann Boundary Problem on Riemann Surfaces (Mathematics and its Applications), Vol. 16, Reidel, Dordrecht, 1988.

[41] E. B. Saff, V. Totik, Logarithmic potentials with external fields, Vol. 316 of Grundlehren der Mathematischen Wissenschaften. Springer, Berlin, 1997.

[42] M. Sakai, Quadrature Domains, Lect. Notes Math. 934, Springer-Verlag, BerlinHeidelberg, 1982.

[43] G. Segal, G. Wilson, Loop groups and equations of KdV type. Publications Mathematiques de l'IHES, 61 (1985), p. 5-65

[44] J.-P. Serre, Un théorème de dualité, Comm. Math. Helv. 29 (1955), 9-26.

[45] J.-P. Serre, Algebraic groups and class fields, Graduate Texts in Mathematics, vol. 117, Springer, Berlin, 1988.

[46] H. S. Shapiro, The Schwarz function and its generalization to higher dimensions, Uni. of Arkansas Lect. Notes Math. Vol. 9, Wiley, New York, 1992.

[47] B. Simon, Orthogonal polynomials on the unit circle. Part 1. Classical theory. AMS Colloquium Publ., 54, Part 1. AMS, Providence, RI, 2005.

[48] R. P. Stanley, Enumerative combinatoric, Vol. 2, Cambridge University Press, 1999.

[49] B. Sturmfels, Introduction to resultants, in: D. Cox, B. Sturmfels (eds.), Applications of Computational Algebraic Geometry, Proceedings of Symp. in Applied Math., 53, American Mathematical Society, 1997, pp. 25-39.

[50] J.J. Sylvester, Note on elimination. Philosophical Magazine XVII, 1840, 379-380.

[51] J. Tate, Residues of Differentials on Curves, Ann. Scient. Éc. Norm. Sup. 1968, 4a série, 1, 149-159.

[52] A. K. Tsikh, Multidimensional residues and their applications, Vol. 103 of Translations of Mathematical Monographs. AMS, Providence, R.I., 1992. 
[53] A. N. Varchenko, P. I. Etingof, Why the Boundary of a Round Drop Becomes a Curve of Order Four, American Mathematical Society AMS University Lecture Series, Vol. 3, Providence, Rhode Island 1992.

[54] van der Waerden, Algebra I, Springer-Verlag.

[55] A. Weil, Oeuvres Scientifiques, Springer-Verlag, New York-Heidelberg-Berlin, 1979.

[56] H. Widom, Asymptotic behavior of block Toeplitz matrices and determinants II, Adv. Math., 21 (1976), 1-29.

Mathematical Department, KTH

E-mail address: gbjorn@kth.se

Mathematical Department, Volgograd State University, Current: MatheMATICAL DEPARTMENT, KTH

E-mail address: tkatchev@kth.se 